\title{
Experimental evaluation of methodologies for single transient cavitation bubble generation in liquids
}

\author{
Darjan Podbevšek $^{1}$ [D $\cdot$ Žiga Lokar $^{1} \cdot$ Jure Podobnikar $^{1} \cdot$ Rok Petkovšek $^{1} \cdot$ Matevž Dular $^{1}$
}

Received: 28 March 2021 / Revised: 22 June 2021 / Accepted: 4 July 2021 / Published online: 19 July 2021

(c) The Author(s) 2021

\begin{abstract}
Single bubble dynamics are of fundamental importance for understanding the underlying mechanisms in liquid-vapor transition phenomenon known as cavitation. In the past years, numerous studies were published and results were extrapolated from one technique to another and further on to "real-world" cavitation. In the present paper, we highlight the issues of using various experimental approaches to study the cavitation bubble phenomenon and its effects. We scrutinize the transients bubble generation mechanisms behind tension-based and energy deposition-based techniques and overview the physics behind the bubble production. Four vapor bubble generation methods, which are most commonly used in single bubble research, are directly compared in this study: the pulsed laser technique, a high- and low-voltage spark discharge and the tube arrest method. Important modifications to the experimental techniques are implemented, demonstrating improvement of the bubble production range, control and repeatability. Results are compared to other similar techniques from the literature, and an extensive report on the topic is given in the scope of this work. Simple-to-implement techniques are presented and categorized herein, in order to help with future experimental design. Repeatability and sphericity of the produced bubbles are examined, as well as a comprehensive overview on the subject, listing the bubble production range and highlighting the attributes and limitation for the transient cavitation bubble techniques.
\end{abstract}

Darjan Podbevšek

darjan.podbevsek@fs.uni-lj.si

1 Faculty of Mechanical Engineering, University of Ljubljana, Askerčeva 6, 1000 Ljubljana, SI, Slovenia 


\section{Graphic abstract}

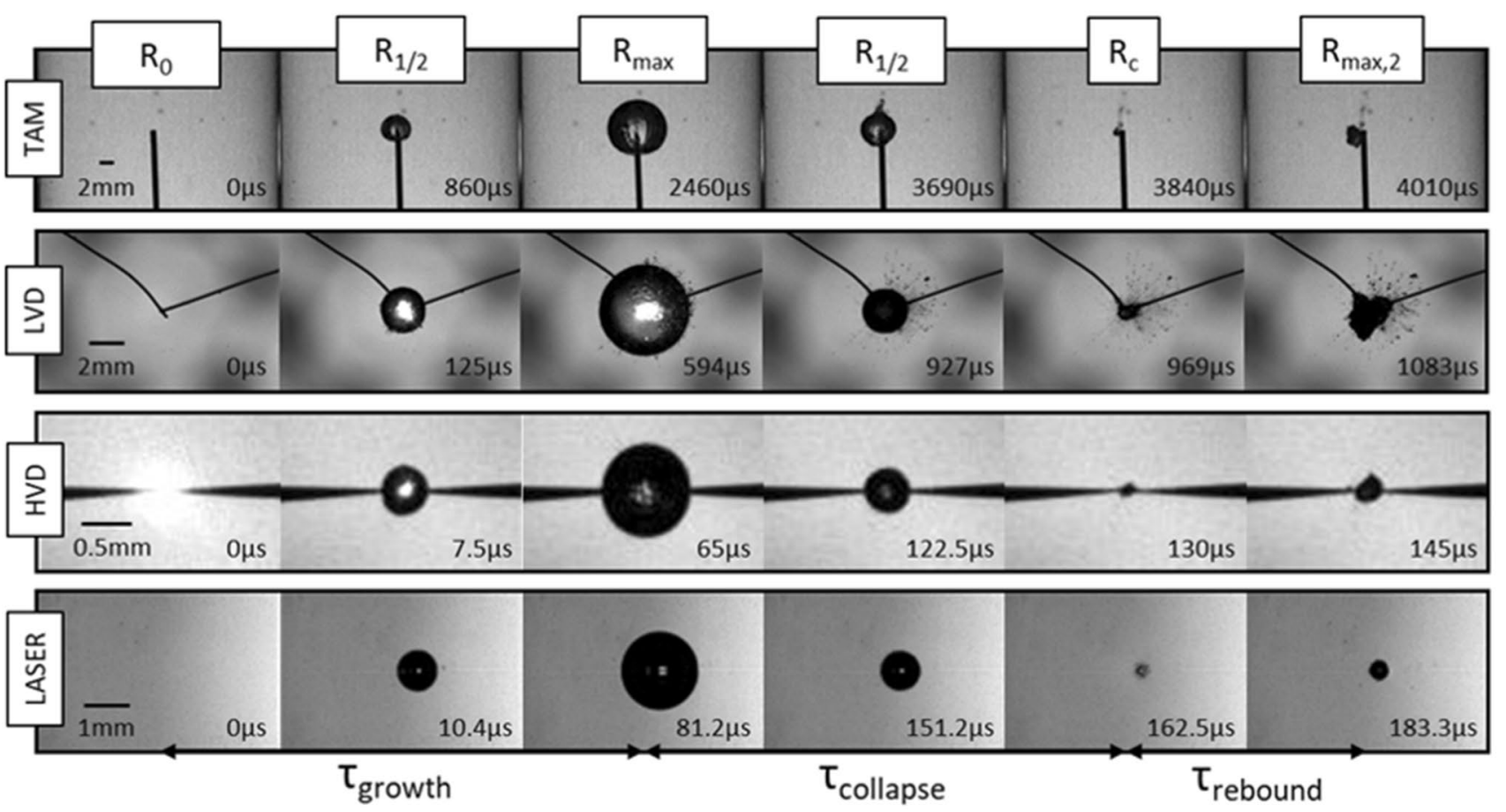

\section{Introduction}

The phenomenon known as cavitation is the emergence of vaporous voids in the liquid as its intermolecular cohesive bonds are overcome. It is often generalized as the appearance of bubbles in the liquid when the pressure drops below liquid vapor pressure. In practice, it often also accounts for the accompanying degassing and the expansion of already present uncondensed gas nuclei in the liquid (Trevena 1984), as the two are hard to dissociate; however, thermodynamically, cavitation is defined as the phase change from liquid to vapor. It is a well-known phenomenon in fluid dynamics, as the phase transition is one of the limiting factors in fluid transport. At the beginning of the twentieth century, while studying ship propeller design, observations of cavities forming at the wake of the propeller were linked to the drastically lower efficiency of the propulsion system (F.R.S 1917). Since then, it was found that dynamics of these bubbles are responsible for many unwanted, as well as beneficial effects of cavitation, which in recent years started attracting ever greater attention in scientific and engineering fields. The former range from mild effects such as noise emissions and vibrations, to efficiency drop and even erosion of solid walls (Dular and Petkovšek 2015; Luo et al. 2016; Dular et al. 2019), while positive effects are numerous, stemming from the inherent energy focusing properties of cavitation bubble dynamics. These have applications from chemical (Zupanc et al. 2014; Dular et al. 2016; Gągol et al. 2018) and biological (Šarc et al. 2016; Kosel et al. 2017; Zupanc et al. 2019) wastewater treatment, material productions (Qiu et al. 2019), cleaning (Verhaagen and Fernández Rivas 2016), process intensification (Sajjadi et al. 2015; Zhang et al. 2016b), to a wide field of fundamental research in physics (Azouzi et al. 2013), chemistry (Grieser et al. 2015; Nikitenko and Pflieger 2017; Podbevsek et al. 2018; Podbevšek et al. 2021), biology (Patek and Caldwell 2005; Iosilevskii and Weihs 2008; Vilagrosa et al. 2012) and medicine (Stride and Coussios 2010).

Cavitation can be broadly grouped into two categories based on excitation (Young 1999): tension and energy deposition methods. The former relies on a low-pressure wave or a region in the flow to provide the driving force for the vapor bubble growth, which is typical for hydrodynamic and acoustic cavitation, whereas the latter occurs by energy deposition from elementary particles (electrical current (Sato et al. 2013), photons (Sato et al. 2013; Padilla-Martinez et al. 2014), neutrons (Taleyarkhan et al. 2002), protons (Futakawa et al. 2014)), driving nucleation and cavitation bubble growth. Often single transient bubbles are studied as the elementary structures in complex multi-bubble or 
multi-scale phenomena, encountered in cavitation research, as their dynamics are often key to eliciting the governing principles of the phenomena. A single bubble in an acoustic field, where the bubbles undergo multiple (few to several thousands) growth/collapse cycles, has been a topic of much research (Crum 2015; Yasui 2018), most notably the single bubble sonoluminescent studies, where the periodic oscillations generate plasma and light emission at peak collapse (Suslick and Flannigan 2008). However, often the aim is to study a single growth/collapse event of a cavitation bubble, for which periodic acoustic, as well as hydrodynamics excitation are ill suited. When using acoustic excitation, it is improbable that a single transient bubble event will be generated. With pulsed acoustic sources, even when tightly focused, a cavitation cloud tends to appear, hindering single bubble observations and also perturbing bubble dynamics due to bubble-bubble interactions and reflected sound waves. On the other hand, single bubbles can be held in place by standing waves and used to observe stable oscillating acoustic bubbles. The acoustic geometry will determine the antinode positions, which are used for acoustic trapping of the introduced gas bubbles, making it difficult to manipulate and position in an experimental setting. Overall, the stable oscillating bubbles generated by acoustic excitation do not represent the dynamics of transient bubbles often encountered in cavitation studies, like nuclei in the liquid exposed to pulsed pressure variations such as shockwaves. Transient bubbles are important for studying, bubble-bubble, bubble-interface and bubble-solid interactions, relevant for disinfection studies of microbiological (Lajoinie et al. 2016) and biological tissue interactions (Vogel and Venugopalan 2003), enhanced mixing at small scales (Hellman et al. 2007), medical applications (Mohammadzadeh et al. 2016), cavitation emulsification (Orthaber et al. 2020), erosion (Dular et al. 2019), cleaning (Song et al. 2004) and thermal effects (Dular and Coutier-Delgosha 2013). Outside the cavitation field, they are interesting for plasma in liquids (Horikoshi and Serpone 2017), acoustic emitters (Buogo et al. 2009), high-voltage breakdown of liquids (Pongrác et al. 2019) and pulsed laser ablation in liquids (Reich et al. 2017) studies.

A single cavitation bubble is an elemental feature of many two-phase flows. Understanding its inception and subsequent dynamics is paramount to explaining many downstream effects in hydrodynamic cavitating flows. Recognizing the importance of transient single bubbles for fundamental studies, researches early on developed several different bubble generation methods. Much of the groundwork was performed during the Second World War, where underwater blast waves were extensively studied (Trevena 1984). The first study using the tube arrest method, dating back to 1952 by W. D. Chesterman (Chesterman 1952). The same year, M. Harrison (Harrison 1952) found similarities between Venturi nozzle-generated and the spark-induced cavitation bubbles. Laser-induced cavities came along sometime later, first reported in 1963, by Askar'yan et al. (Askar'yan et al. 1963), with the development of pulsed ruby laser sources. Although updated, the underlying principle of the three ideas remains, allowing for the methods to be used to this day. In cavitation studies, it is often not a straight forward path to elicit the underlying principles behind the observed effects. The Rayleigh-Plesset equation (RPE) is frequently employed to make sense of the bubble dynamics, as it describes the empty void radius evolution in time (with several assumptions) (Young 1999). With the multitude of parameters influencing cavitation and associated phenomena, it is often of great importance to understand mechanism at play on a single bubble level. It is for this reason that a robust generation of repeatable and controllable single vapor bubbles is of fundamental significance when studying cavitation. In practice, several methods are used, but not often enough is there the discussion of the suitability of the method for the particular question raised, nor are there many cross-comparison or reviews covering this field. As studies involving transient vapor bubbles are of interest in an ever-broader and often specialized fields, an informed decision on the experimental setup is not always straightforward. It is hard to expect everyone studying these phenomena, especially when starting out, to grasp all the different underlying physics behind various vapor bubble generation at our disposal. Moreover, it is not uncommon to question whether the experimental setup and the generation method affects the observed results of the observed phenomenon. For such instances it is useful to know alternative methods available to the user, along with their attributes and limitations.

Besides the advancement of the state of the art in experimental techniques, which will be elaborated in "Experimental setup" section, we wish to address the issues of using various experimental approaches to study the single cavitation bubble phenomenon and its associated effects. In past years, numerous studies were published on the topic, with results extrapolated between technique and on to "realworld" cavitation. We show that each technique has its pros and cons, and the results can be useful for understanding various aspects of the cavitation phenomenon; however, one still needs to be careful with interpretations, as some features of cavitating flows are poorly represented with bubbles generated by certain methods. For this reason, we compared the most commonly used transient single spherical bubble generation techniques: the tube arrest method (TAM), the laser-induced method and the high- (HVD) and low-voltage electrical discharge (LVD). The pulsed laser-induced bubble generation is considered a reference technique to which the other three are compared. An overview as well as the chain of events leading to bubble generation is discussed for each family of techniques in the theoretical background section. 
Several modifications were implemented for the TAM, HVD and LVD experimental setups, allowing for safer, repeatable and tuneable bubbles to be produced. Critical parameters from previous studies are catalogued in a user-friendly manner for all four discussed methods. A comparative study on all techniques is presented in results, focusing on the governing parameters, sphericity of the produced bubbles and their repeatability, while bubble lifetimes are compared to the Rayleigh model. The techniques compared herein, present practical and inexpensive experimental setups for single transient bubble generation. At the end, we discuss the attributes, limitation and applicability for the techniques herein and other techniques not in the scope of the study.

\section{Metodological background}

The division of single cavitation bubble generation is shown in Fig. 1. Tension-induced methods can be subdivided in compression reflected and direct tension-based excitation, while energy deposition techniques split into electrical discharge and optical techniques. Techniques marked with the asterisk are used in this work.

\subsection{Tension-induced techniques}

Tension-induced techniques, as the name suggest, rely on tensional stress being applied to the liquid, inducing nucleation and bubble growth. Static techniques like the Berthelot tube (Jones et al. 1981; Overton et al. 1982) and centrifugation techniques (Briggs 1950) apply tensile stress to the liquid by isochoric cooling and centrifugal force, respectively. Along with the quartz inclusions, pull technique (mechanical bellows) and liquid superheating techniques, were widely used in the study of water metastability and its tensile strength (Caupin and Herbert 2006). Dynamic techniques were also used to this end; however, since they rely on density fluctuations or shock waves to perturbate the liquid from its equilibrium state, it is not always easy to determine the cavitation threshold, as pinpointing when a cavitation event has occurred can be difficult. Eventually, the theoretically predicted homogeneous cavitation limit was reached with isochoric cooling of water in quartz inclusions (Zheng et al. 1991; Azouzi et al. 2013). On the other hand, the fact that most important cavitation phenomena originate from dynamic tensional stressing (Williams and Williams 2002) and owing to their simple design, dynamic methods remain the preferred choice for cavitation bubble generation. A compression wave is in practice easier to produce, compared to a tension wave; however, high-pressure shock waves are typically followed by a tension wave (Ohl 2002a; Ohl and Ikink 2003), which can (as in lithotripters), induce cavitation events (Arora et al. 2007; Ikeda et al. 2016). Another way to generate cavitation from high-pressure surges is to reflect it from a free surface (Ando et al. 2012) or from a medium acoustic impedance mismatch, causing a phase reversal and the reflected tensile wave. This is also the principle for the well-known phenomenon of nucleation and subsequent foaming of beer (Rodríguez-Rodríguez et al. 2014), as it is hit on top of the glass bottle. The compression wave travels to the bottom of the bottle where it is transferred to the liquid as a tension wave, inducing cavitation bubbles (RodríguezRodríguez et al. 2014), and for non-gas-saturated liquids, it can also cause bottle breaking, due to intense cavitation bubble collapse (Daily et al. 2014).

The drawback of compression wave-driven techniques is the initially compression or even elimination of the nuclei, which could affect the bubble dynamics later on (Andersen and Mørch 2015). For this reason, the tube arrest method with the "ab initio" tension wave seems better suited, which

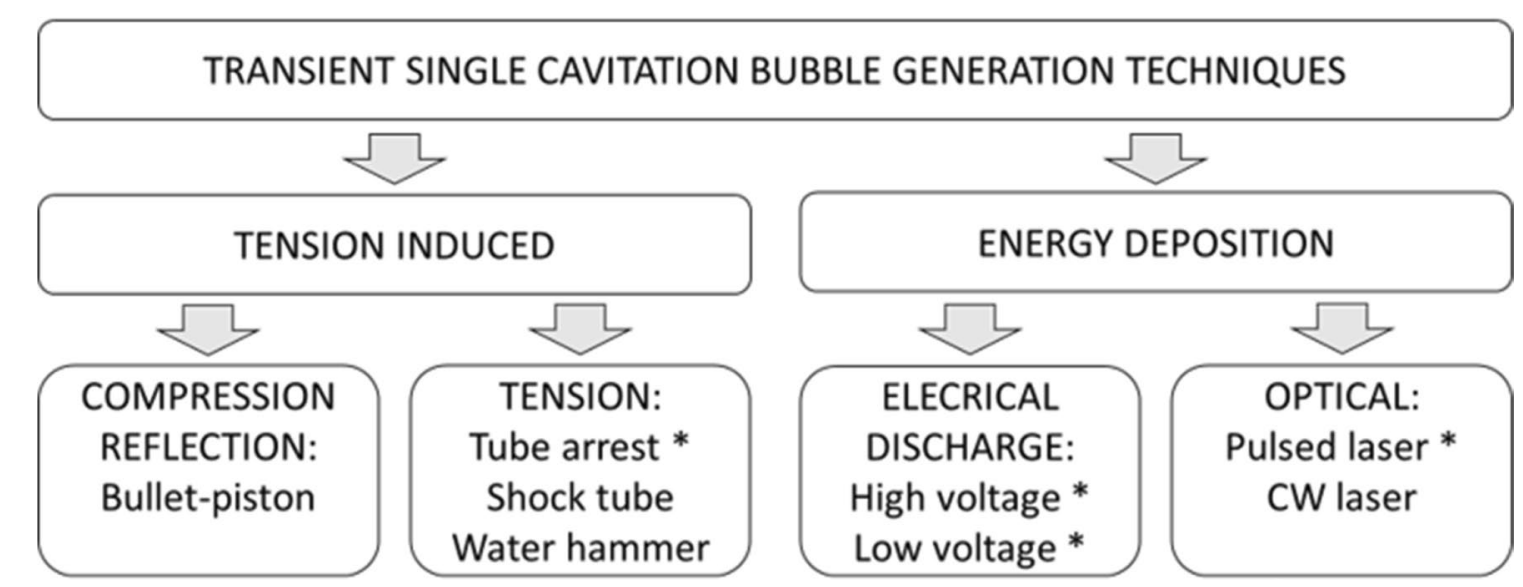

Fig. 1 Classification of transient cavitation bubble generation techniques. Methods further discussed in the article are marked with an asterisk (*) 
more accurately mimics real-world situations. Another thing to consider is the bubble collapses in shock wave techniques can deviate from the theoretical Rayleigh-like collapse, where the pressure changes around the bubble are instantaneous and homogeneous (Kapahi et al. 2015). This can also produce jetting in the direction of the wave propagation $(\mathrm{Ohl}$ and Ikink 2003, p.). Nonetheless, techniques like the bullet piston (Williams et al. 1998; Williams and Williams 2002; Williams * and Williams 2004), water shock tube (Richards et al. 1980) and glass bottle or test tube impact (Kiyama et al. 2015; Pan et al. 2017) have been used in cavitation research. An interesting approach for studying cavitation nucleation under transient pressures used a gravity-driven device capable of creating compression tension or just tension excitation waves (Andersen and Mørch 2015). Water shock tube can produce tension waves by reflection (Richards et al. 1980) or directly (Fujikawa and Akamatsu 1978), depending on the setup. It relies on a membrane rupture to create a pressure discontinuity (step change), driving cavitation. Another technique is called the water hammer (alternative nomination vibrating liquid column (Buchanan et al. 1962; Baird 1963)) technique. As the name suggests, it is based on the water hammer effect, encountered with fast valve shutting and the consequent liquid mass arrest, which is problematic in liquid transport, stressing hydraulic elements and piping (Bergant et al. 2006). The water hammer apparatus shakes a cylindrical tube filled with fluid. The periodic direction changes induce up to $2 \mathrm{~g}$ of acceleration, forming tensional stress (by reflection from free surface) in the liquid (Su et al. 2003). With bubbles typically larger than acoustic cavitation and periodic production unlike typical tension-induced techniques, its main drawback is poor repeatability and non-localized bubble production between cycles (Su et al. 2003). Recently, a modified water hammer technique is used to enhance the collapse of a laser-induced bubble, with the aim of enhancing the collapse intensity (Rosselló et al. 2018).

The tube arrest method (TAM) remains one of the more popular transient bubble generation techniques, due to its simplicity and the "ab initio" tension wave as the driving force as opposed to techniques relying on reflecting compression waves from a free surface, i.e., bullet piston method (Williams et al. 1998). It relies on the inertia of an upward moving volume of liquid to induce tensional stress, when its container is brought to an abrupt stop. The principle of generation of a low-pressure (tension) wave with TAM is shown in Fig. 2. A strong mounting frame (a) holds and guides the rigid tube (b), allowing only one-dimensional movement. The tube typical made of transparent material (glass, plexiglass or polycarbonate) contains the liquid and the nucleation site (c). The propulsion is provided by the spring (d); as it gets compressed (1) and released (2), it thrusts the liquid vessel toward a fixed rigid boundary (e). The impact halts the vessel almost instantaneously (3), while the liquid inside retains its momentum. This creates a tension wave (3), starting from the bottom of the vessel, moving toward the top, diving the bubble growth (4) and collapse (5), with subsequent rebounds. Eventually, depending on the experimental design, the phase reversal of the excitation wave can occur, producing compression waves in the system. There are many forceable ways to induce motion of the tube, but a spring is often chosen due to its simplicity. However, this can cause repeatability issue and unwanted rebound of the tube. Relatively large bubbles $(>5 \mathrm{~cm}$ ) can be produced, with according growth and collapse times (ms). The tension wave generated is determined by the deceleration achieved at
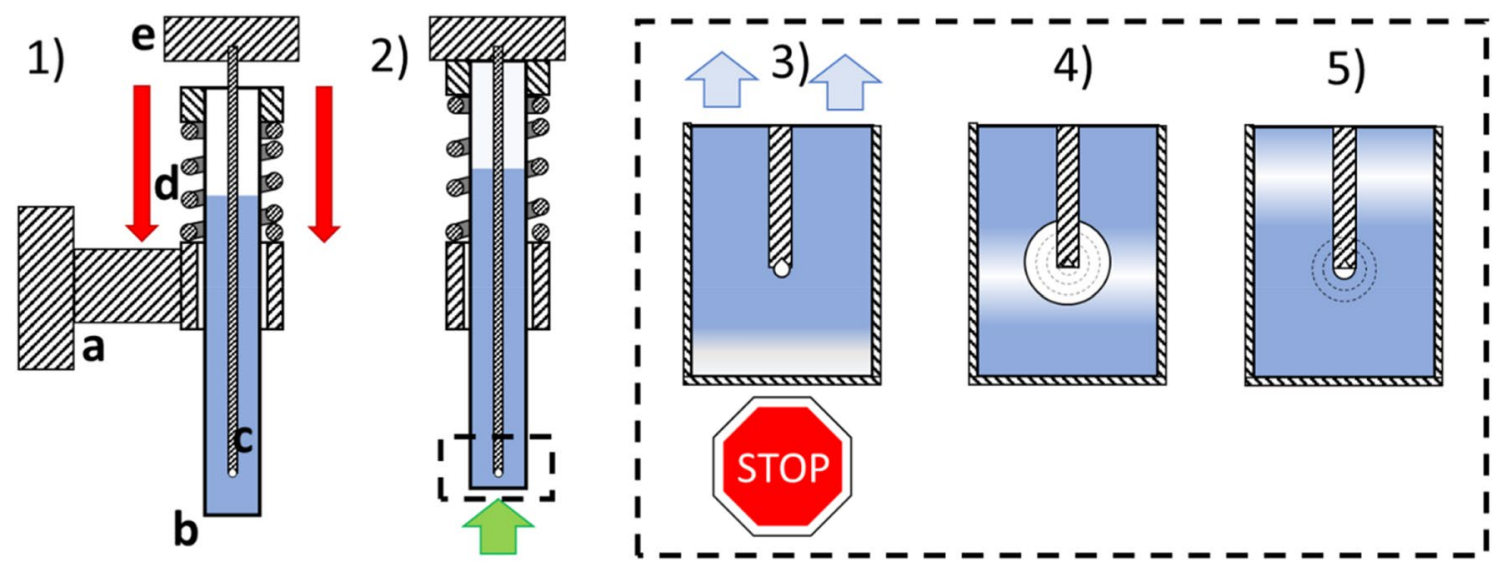

Fig. 2 Tube arrest method (TAM) operation principle. The rigid mounts a hold the tube with the liquid $\mathbf{b}$ and the rod with the nucleation bubble $\mathbf{c}$ in place, while the spring $\mathbf{d}$ is used to propel it toward the upper rigid mount $\mathbf{e}$. The spring is compressed (1) and released (2), propelling the tube with the liquid. As it is abruptly stopped (3), a tension wave (white region) is formed by the liquid upward momentum. The tensile wave moves up the tube to the nucleation site at the end of the inception rod, driving the cavitation bubble growth (4). The collapse occurs as the pressure normalizes (5) 
impact; therefore, the arrest should be as abrupt as possible to induce a high-amplitude tension wave.

A comprehensive overview of parameters for the TAM obtained from the literature are presented in Table 1. The tube diameters used range between 1 (Chesterman 1952) and $3 \mathrm{~cm}$ (Dular and Coutier-Delgosha 2013), roughly $1 \mathrm{~m}$ in length, made either of glass (Chesterman 1952; Qi-Dai and Long 2004; CHEN Qi-Dai and CHEN Qi-Dai 2004; Wu et al. 2005; Chen and Wang 2005; Chong-Fu et al. 2008) or more commonly polycarbonate (Williams et al. 1997a, b, 1998; Williams P. R. et al. 1999) or plexiglass (Schmid 1959; Dular and Coutier-Delgosha 2013; Andersen and Mørch 2015), as they are less brittle. A sufficient tube size should be used to allow for the bubble to develop without the vessel wall interference. Generated bubbles are between 1 and $6 \mathrm{~cm}$ (Schmid 1959; Dular and Coutier-Delgosha 2013), and the tube diameter should be roughly $5 \mathrm{~cm}$, to avoid wall interaction (Chen and Wang 2005). The end velocities of the tube $0.5-6 \mathrm{~m} / \mathrm{s}$ (Chesterman 1952) with the useful range usually below $2 \mathrm{~m} / \mathrm{s}$ (Chesterman 1952; Dular and CoutierDelgosha 2013), as glass tube tend to break and bubbles get distorted above this values (Overton et al. 1984). Distilled or tap water is used, sometimes degassed in attempt to make as vaporous bubbles as possible (Dular and Coutier-Delgosha 2013). A few studies used glycerin (CHEN Qi-Dai and CHEN Qi-Dai 2004), lubrication oils (Williams et al. 1997b) and kerosene (Overton et al. 1984) as the working fluid. The design usually includes an inception bubble between 0,5 (Schmid 1959; Qi-Dai and Long 2004; CHEN Qi-Dai and CHEN Qi-Dai 2004; Wu et al. 2005; Chen and Wang 2005) and $2 \mathrm{~mm}$ (Dular and Coutier-Delgosha 2013) in diameter, commonly at the end of a metallic tube (few mm diameter), or depending on the experiment, and the nucleation can be generated without a prefixed point (Chesterman 1952; Overton et al. 1984; Williams et al. 1997a; Williams P. R. et al. 1999), usually manifesting as a cluster of cavitation bubbles $1-2 \mathrm{~cm}$ above the cylinder base (Trevena 1984). Some induce nucleation bubbles which float upwards due to buoyancy and trigger the apparatus when the bubble floats past the desired point (Williams et al. 1997b). The tube travel distance is usually in the range between 10 and $20 \mathrm{~mm}$ (Dular and Coutier-Delgosha 2013), but can go up to $80 \mathrm{~mm}$ (Wu et al. 2005), depending on the design. The nucleation rod is suspended from the top of the apparatus, not in contact with the tube. This leads to a relative difference in velocity of the liquid and the stationary rod, which can deform the bubble shape after the arrest and bubble growth are initiated.

In "Experimental setup" section, we demonstrate the tube arrest method driven by pneumatic cylinders instead of a spring. To our knowledge, this is the first reported case of such a device, with several other improvements implemented as well.

Table 1 Overview of parameters for cavitation bubble generated by the TAM (tube arrest method), from different sources. Data marked with asterisk $(*)$ are not provided by the authors of the reference, but are approximated from the corresponding article for comparison

\begin{tabular}{|c|c|c|c|c|c|}
\hline Reference & $\begin{array}{l}\text { Nucleation bubble } \\
\text { size }[\mu \mathrm{m}] \text { and } \\
\text { liquid }\end{array}$ & $\begin{array}{l}\text { Internal tube } \\
\text { diameter }[\mathrm{m}] \\
\text { and material }\end{array}$ & Liquid height $[\mathrm{m}] /$ mass $[\mathrm{g}]$ & $\begin{array}{l}\text { Bubble size }[\mathrm{mm}] / \text { lifetime } \\
{[\mathrm{ms}]}\end{array}$ & End velocity $[\mathrm{m} / \mathrm{s}]$ \\
\hline (Chesterman 1952) & $\begin{array}{l}>100 \\
\text { water }\end{array}$ & $\begin{array}{l}0.0115 \\
\text { Glass }\end{array}$ & $0.5 / 51.9$ & $0.5-5 / 2-5$ & $2-6$ \\
\hline (Schmid 1959) & water & $\begin{array}{l}0.032 \\
\text { Acrylic glass }\end{array}$ & $0.4 / 1590$ & $4-8 / 1.7-4,7$ & - \\
\hline (Williams et al. 1998) & $\begin{array}{l}>0.2 \\
\text { water }\end{array}$ & $\begin{array}{l}0.021 \\
\text { PC }\end{array}$ & $0.2-1 / 83-415$ & $-/ 16$ & - \\
\hline (Qi-Dai and Long 2004) & $\begin{array}{l}1000 \\
\text { water }\end{array}$ & $\begin{array}{l}0.026 \\
\text { Glass }\end{array}$ & $0.6 / 317$ & $\sim 16.5 / 30^{*}$ & $1.3-2.8$ \\
\hline (Chong-Fu et al. 2008) & $\begin{array}{l}>0.3 \\
\text { various }\end{array}$ & $\begin{array}{l}0.025 \\
\text { Glass }\end{array}$ & - & $15,4 / 6$ & - \\
\hline $\begin{array}{l}\text { (CHEN Qi-Dai and CHEN } \\
\text { Qi-Dai 2004) }\end{array}$ & $\begin{array}{l}1000 \\
\text { glycerin }\end{array}$ & $\begin{array}{l}0.026 \\
\text { Glass }\end{array}$ & $0.6 / 318$ & $3,6-17 / 7-26^{*}$ & $0.8-3.0$ \\
\hline $\begin{array}{l}\text { (Dular and Coutier-Delgo- } \\
\text { sha 2013) }\end{array}$ & $\begin{array}{l}500 \\
\text { water }\end{array}$ & $\begin{array}{l}0.03 \\
\text { Acrylic glass }\end{array}$ & $0.7 / 495$ & $20 / 15-19$ & $0.5-2$ \\
\hline (Williams P. R. et al. 1999) & $\begin{array}{l}<0.2 \\
\text { water }\end{array}$ & $\begin{array}{l}0.021 \\
\text { PC }\end{array}$ & $1 / 1385$ & $-/ 1.2$ & - \\
\hline (Williams et al. 1997a) & $\begin{array}{l}- \\
\text { water }\end{array}$ & $\begin{array}{l}0.021 \\
\text { PC }\end{array}$ & $0.5 / 172$ & $7.5 /-$ & - \\
\hline (Wu et al. 2005) & $\begin{array}{l}1000 \\
\text { various }\end{array}$ & $\begin{array}{l}0.027 \\
\text { Glass }\end{array}$ & $0.5 /-$ & $20-25 / \sim 10$ & 1.7 \\
\hline (Chen and Wang 2005) & $\begin{array}{l}1000 \\
\text { water }\end{array}$ & $\begin{array}{l}0.026 \\
\text { Glass }\end{array}$ & $0.7 / 370$ & $17.5-32 / 13.2-33.6$ & $1.2-2.8$ \\
\hline
\end{tabular}




\subsection{Energy deposition techniques}

Generation of vaporous voids with energy deposition can be achieved with electrical discharge or optical techniques. The former is the consequence of high electrical fields in the liquid (Avila et al. 2015) and the latter optical breakdown and/ or stress confinement of the liquid (Quinto-Su et al. 2008). Stress confinement predicts the energy deposition will be much faster than the mechanical or thermal relaxation in the system, as is the optical breakdown caused by absorption at the focal point. With both techniques, the initial pulsed energy deposition onto a small focused volume produces hot non-thermal plasma in the liquid (Jiang et al. 2014; Lazic and Jovićević 2014; Horikoshi and Serpone 2017), which drives the bubble nucleation and growth. Plasma generation in liquids is itself a dynamic, transient and complex process still under investigation (Bruggeman et al. 2016). Plasma expansion in dense, weakly compressible media (such as liquids) is inhibited, causing higher temperatures and pressure than in compressible gaseous media. Therefore, for a liquid environment, there is a tendency for the energy to be transferred into mechanical energy, so the creation of compression wave, followed by a tension trailing wave, leads to vapor bubble generation (Lazic and Jovićević 2014). As the excited electrons create hot non-thermal plasma, the following expansion of the hot plasma/gas bubble (Yan and Chrisey 2012; Lazic and Jovićević 2014) is similar for laserand spark-driven techniques (Sato et al. 2013). At $R_{\max }$, the bubble reaches thermodynamic equilibrium and follows the theoretical collapse dynamics of an empty void (Sato et al. 2013). As the plasma is generally hard to produce in liquids, both techniques are interesting also from the point of view of plasma research (Lazic and Jovićević 2014). Bubble rebounds typical for plasma-induced bubbles indicate that some of the content is uncondensed gas, either oxygen and hydrogen from water splitting or degassed gas from the liquid during growth (Lew et al. 2007). Several rebounds are typical for this type of generation. However, compared to ultrasound sources, $\mathrm{HV}$ arc discharge-generated bubbles have the advantage of producing bubbles mostly made of vapor (Buogo and Cannelli 2002). This should hold also for laser-induced bubbles, as the underlying principle for their generation is similar, at least from a fluid dynamics standpoint.

\subsubsection{Electrical discharge}

Underwater spark discharges are used in various applications, ranging from environmental (Locke 2012; Jiang et al. 2014), nanomaterial production (Chen et al. 2015), medicine (Sunka et al. 2004), high-voltage transformers or switches (Lewis 1994) and bubble dynamics studies (Vokurka 1988; Buogo et al. 2009). Due to its simple application, it is a popular method for inducing an impulsive sound source, as a spherical bubble will nicely approximate a zeroth-order acoustic radiator (Buogo et al. 2009), making it interesting for deep sea prospecting (Cannelli et al. 1990), minesweeping (Fry et al. 1999), oceanic seismic exploration (Sun et al. 2009). Essentially there are three parts to the process: the pre-breakdown streamer formation $(\sim \mathrm{ns})$, the spark discharge (ns to $\leq \mathrm{ms}$ ) and the following bubble growth and collapse ( $\mu$ s to ms) (Buogo et al. 2009). The pre-breakdown period, which is the time the streamers span the electrode gap and before the spark production in the vapor channel (Rond et al. 2018). It is a subject of research for decades, with implications in high-voltage transformers and switches, as the mechanism of initiation and propagation for this process are still not well understood.

A typical spark-induced cavitation setup will include two electrodes submerged in a liquid. Commonly a capacitor discharge is introduced over the electrodes, establishing a conductive channel between them filled with non-thermal plasma (Jiang et al. 2014; Horikoshi and Serpone 2017). As the plasma channel heats up, an intense current will flow through the channel causing extreme local heating and subsequent evaporation and vapor bubble growth (Vokurka 1988). Electrical discharge techniques can be roughly divided into high- and low-voltage methods, each having their pro and cons.

2.2.1.1 High-voltage discharge A first rough categorization of high-voltage-induced bubbles can be by either coronalike or arc discharge. A very fast (sub-ns) HVD (highvoltage discharge) can in fact lead to corona-like discharge (considered a partial discharge (Bruggeman et al. 2016)) without the formation of a bubble (Pongrác et al. 2019), as the bubble growth is usually in the $\mu$ s range and the dissipation energy in liquids is relatively quick. The pre-breakdown phase change is believed to originate from nanosized voids created by electrostriction effect of the local high electric fields needed to create and sustain an electronic avalanche in liquids. However, coronal discharge has gained interest as a cost-effective alternative to pulsed arc discharge for oceanic seismic exploration and hydro-acoustic research (Huang et al. 2014). Bubble generation in this case is an electrothermal effect by Joule heating (Huang et al. 2015). Plasma generation can occur at either anode, cathode or both electrodes. As the water around the tip evaporates due to the high-density current at the electrode tip, the remaining energy will go toward plasma generation in the vapor or at the gas-liquid interface (Huang et al. 2014). The electrode configuration is often a pin to plate, with the plate being the conductive metallic housing and a sharp needle acting as the pin, around which high electric fields will form the coronalike discharge (Jiang et al. 2014). Similarly, the pulsed arc discharge is initiated by a highly non-uniform el. field at the 
electrode tips. A pre-breakdown cavity is formed by a combination of Joule heating of the liquid (Atrazhev et al. 2010), electron emission (Atrazhev et al. 2010) and electromechanical rupture of the liquid (Lewis 1996). The low permittivity of the gaseous void and the high electrical field therein trigger the ionization process at the gas-liquid interface (Sun et al. 2016). Plasma streamers extending to the bulk liquid and onto the opposite electrode bridge the interelectrode gap and which develops into a plasma channel (Timoshkin et al. 2006). The time-varying resistance of the plasma channel determines the temporal energy dissipation in the cavity (Timoshkin et al. 2006). The relatively low temperature of the plasma leads to an initially high resistance, dropping as it heats up. Rapidly the energy is deposited into the plasma channel, leading to high temperatures and pressure of the highly conductive ionized gas. The explosive expansion can generate pressure waves. As the bubble is assumed to reach thermodynamic equilibrium at maximum radius (Sato et al. 2013), the subsequent collapse caused by the hydrostatic pressure is said to mimic the Rayleigh collapse for the empty void (Buogo and Cannelli 2002). An estimation of the transferred energy to the vapor bubble can be calculated from the $\mathrm{R}_{\max }$ (bubble maximum radius) (Buogo et al. 2009; Sato et al. 2013; Zhang et al. 2016a), with the equation $E_{b}=\frac{4}{3} \pi p_{0} R_{\max }^{3}$, with $\mathrm{p}_{0}$ being the ambient pressure. Typical parameters used in HVD bubble techniques are listed in Table 2, reviewing arc and coronal discharges. Often the high-voltage source will charge a small capacitor to provide a pulsed discharge. An alternative low-energy setup uses a simple $2.3 \mathrm{kV}$ piezoelectric spark discharge from a lighter to induce a hemispherical vapor bubble by (Avila et al. 2015; Gonzalez-Avila et al. 2020). In our experiment, we used a larger, $16 \mathrm{kV}$ piezoelectric spark generator and tungsten electrodes, demonstrating that a reliable, safe and cheap spherical bubble generator can be obtained. Similar to the pulsed laser-induced bubble, the underlying principles for bubble production is stress confinement, as the fast energy deposition creates driving pressure waves.

2.2.1.2 Low-voltage discharge As the name suggest, the LVD (low-voltage discharge) uses a voltage range that is much safer for the operator $(<60 \mathrm{~V})$, and is a preferred bubble generation technique for several authors (Turangan et al. 2006; Lew et al. 2007; Khoo et al. 2009; Fong et al. 2009; Pain et al. 2012; Gong et al. 2012; Goh et al. 2013; Luo et al. 2018). In the low-voltage discharge case, the electrodes in contact essentially create a scenario where the highest resistivity of the circuit is at the electrode contact, due to the smallest conducting cross section. The dissipated energy from the capacitor discharge $-\mathrm{E}_{\mathrm{c}}-$ creates the spark, which in turn causes heating and vaporization, expanding the bubble to due to the high internal pressure and temperature. This is a highly energetic event which frequently causes fragmen- tation or breaking of the electrodes, needing repositioning after each discharge, which may lead to inconsistencies if not properly set up. The discharge event is much longer than that with $\mathrm{HV}$ and is usually visually present during much of the bubble growth, which can interfere with observations, especially during the initial phase [20]. As the content expands and cools, eventually the inner pressure in the bubble will be far below ambient pressure, due to the inertia of the growing interphase and a collapse due to ambient pressure will ensue. The different parameters used for the LVD from the literature are shown in Table 3. Bubbles range from 2 and $5 \mathrm{~mm}$ with lifetimes of a few $\mathrm{ms}$. The electrodes used are typically copper wires from a typical multi-strand 0.1-0.15-mm-diameter wire, with 30-100 V (Luo et al. 2018) potential, which in the range for a typical laboratory power supply is used to charge the capacitors circuit through a "pull up" resistance of about $1 \mathrm{k} \Omega$. These are commonly a few thousand $\mu \mathrm{F}$. A typical electric circuit for LV electrical discharge technique will encompass a charging, discharging, sparking, storage subsections. The charging and discharging relays are used to fill and empty the capacitor(s) in the storage section, while the sparking section will introduce the stored el. energy to the electrodes and induce a bubble. We use a low-voltage discharge circuit much like the one used in (Goh et al. 2013), described in "Experimental setup" section. Multiple electrode setups exploring bubble interactions have been employed by (Khoo et al. 2009; Fong et al. 2009), for studying bubble-bubble interactions, with and without phase shifted bubble dynamics.

\subsubsection{Optical techniques}

Optical techniques are least intrusive and invasive of all the methods studied, since no electrodes or nucleation sites are needed, and as such are likely to produce homogeneous nucleation. When the laser pulse is focused on a solid-liquid interface, the process is called ablation. The absorption of the laser takes place on the surface, vaporizing material and forming a hemispherical bubble (Lam et al. 2016). An extensive review on pulsed laser ablation in liquids (PLAL) is available at (Yan and Chrisey 2012). Other studies focus on the bubble formation stage of this multi-scale phenomenon and their influence on micro- or nanomaterial production (Lam et al. 2016; Reich et al. 2017). When the light in the bulk liquid, plasma formation via the multi-photon ionization is said to occur with fast (fs) high-photon-density pulsed excitation and with the use of very pure liquids, cascade ionization is more common for longer pulse times (ns) (Lazic and Jovićević 2014). While both are fast enough to initiate the cavitation events, the effect of the different ionizations process is not clear, it should have limited influence beyond inducing stress confinement; the plasma generation is in the fs-ns, while the bubble lifetime usually in $\mu$ s-ms range. 


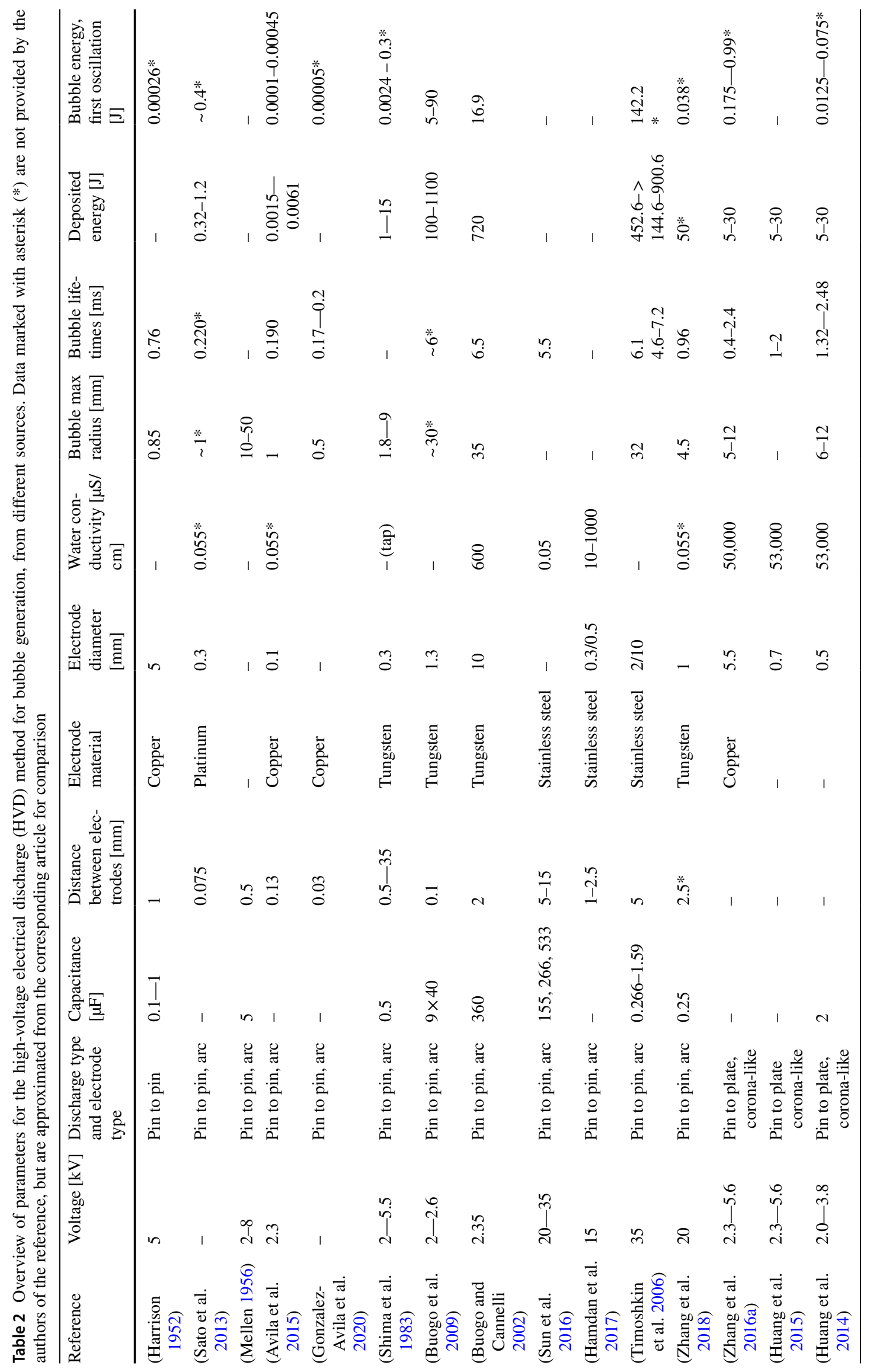


Table 3 Overview of parameters for the low-voltage electrical discharge (LVD) method for bubble generation, from different sources. Data marked with asterisk (*) are not provided by the authors of the reference, but are approximated from the corresponding article for comparison

\begin{tabular}{|c|c|c|c|c|c|c|c|c|}
\hline Reference & Voltage [V] & $\begin{array}{l}\text { Capacitance } \\
{[\mathrm{mF}]}\end{array}$ & $\begin{array}{l}\text { Electrode } \\
\text { diameter } \\
{[\mathrm{mm}]}\end{array}$ & $\begin{array}{l}\text { Electrode mate- } \\
\text { rial }\end{array}$ & $\begin{array}{l}\text { Bubble max } \\
\text { radius [mm] }\end{array}$ & $\begin{array}{l}\text { Bubble } \\
\text { lifetimes } \\
{[\mathrm{ms}]}\end{array}$ & $\begin{array}{l}E_{c}-\text { Deposited } \\
\text { energy }[\mathrm{J}]\end{array}$ & $\begin{array}{l}\mathrm{E}_{\mathrm{b}}-\text { Bubble } \\
\text { energy, first } \\
\text { oscillation }[\mathrm{J}]\end{array}$ \\
\hline $\begin{array}{l}\text { Lew et al. } \\
\text { (2007) }\end{array}$ & 55 & $3.3+2 \times 1.0$ & 0.11 & Copper & $3.6-5.2$ & $1-1.5$ & $0.0275-0.1457^{*}$ & $0.0018-0.0523 *$ \\
\hline $\begin{array}{l}\text { Goh et al. } \\
\text { (2013) }\end{array}$ & 60 & $2.2+4.7$ & 0.1 & Tinned copper & 4.5 & 1.3 & $0.207 *$ & $0.0376^{*}$ \\
\hline $\begin{array}{l}\text { Khoo et al. } \\
\text { (2009) }\end{array}$ & 55 & $3.3+2 \times 1.0$ & 0.11 & Copper alloy & $3-5$ & $1-1.5$ & $0.1457 *$ & $0.0113-0.0523 *$ \\
\hline $\begin{array}{l}\text { Gong et al. } \\
\text { (2012) }\end{array}$ & 60 & 6.9 & 0.1 & Copper & 5 & 1.2 & $0.207 *$ & $0.0523 *$ \\
\hline $\begin{array}{l}\text { Pain et al. } \\
\text { (2012) }\end{array}$ & 60 & 5.5 & 0.5 & - & 5 & $0.8-4.1$ & $0.165^{*}$ & $0.0523^{*}$ \\
\hline $\begin{array}{l}\text { Luo et al. } \\
\text { (2018) }\end{array}$ & $0-100$ & $2 \times 4.0$ & 0.15 & Copper & $5-12$ & $1.2-3.2$ & $0.4^{*}$ & $0.0523-0.724^{*}$ \\
\hline $\begin{array}{l}\text { Turangan et al. } \\
\text { (2006) }\end{array}$ & 55 & $3.3+2 \times 1.0$ & 0.11 & Copper alloy & $2.8-3.5$ & 0.9 & $0.1457 *$ & $0.0092-0.0179 *$ \\
\hline $\begin{array}{l}\text { Fong et al. } \\
\text { (2009) }\end{array}$ & 57 & 3.3 & 0.117 & Copper alloy & $3-5$ & $1-1.1$ & $0.094 *$ & $0.0113-0.0523 *$ \\
\hline $\begin{array}{l}\text { Kannan et al. } \\
\text { (2018) }\end{array}$ & 180 & - & 0.1 & Copper alloy & 12.4 & 3.8 & - & $0.788^{*}$ \\
\hline Xu et al. (2019) & 80 & - & 0.1 & Copper alloy & 8.0 & 2.08 & - & $0.211^{*}$ \\
\hline
\end{tabular}

2.2.2.1 Pulsed laser-induced cavitation The pulsed delivery of photons to the focal spot increases the photon density both temporally and spatially, and facilitates the plasma formation by dielectric breakdown, which initiates homogeneous nucleation of the liquid and makes the pulsed laser a reliable and repeatable bubble generator. Table 4 shows some of the typical parameters from the literature, for pulsed laser-induced cavitation. Some users prefer parabolic mirrors to lenses, as they improve the focus by avoiding aberration and refraction, while achieving high focus angles and with it a compact plasma volume, leading to spherical bubbles (Tinguely et al. 2012; Sinibaldi et al. 2019). However, modern quality optics and low $\mathrm{M}$ numbers of the laser beam profile should minimize or alleviate these problems and allow for much lower laser powers to be used for reliable vapor bubble generation (Table 4). Optical breakdown or thermal stress confinement is usually named as the underlying principles for bubble generation (Quinto-Su et al. 2008). The partition of energy in a collapsing laser-induced bubble was studied by (Vogel et al. 1999; Tinguely et al. 2012). It was found that in microgravity conditions the partition of energy between the shock wave formation and the rebound is governed by a single non-dimensional parameter, involving fluid physical properties, ambient pressure and the noncondensed gas pressure. Moreover, even the hydrostatic difference around the bubble was shown to affect the spherical collapse and cause jetting, for larger bubbles (Supponen et al. 2019). Therefore, even with this method, a perfect, "theory-like" collapse requires special conditions (zero G experiments). Nonetheless, of all our techniques, it is closest to perfect, from a bubble dynamics standpoint, and is therefore considered the benchmark, especially for small bubbles.

2.2.2.2 Photothermal bubbles Thermo-cavitation, photothermal or plasmonic bubbles, all these names are related terms, used for the generation of vapor bubbles by a surface or liquid absorption, reaching spinodal conditions in the liquid (superheating), creating metastable conditions and leading to explosive vaporization. Although this is essentially bringing the liquid to boiling, it is still often termed cavitation as it produces a rapid cavitationlike growth and collapse. The thermo-cavitation phenomenon occurs as a continuous wave (CW) laser is focused in light-absorbing liquids (Padilla-Martinez et al. 2014), instead of more commonly used pulsed laser sources. The technique does not produce plasma, from which the bubble would typically grow, but it locally heats up the liquid to the spinodal limit, making it sensitive to density fluctuations (becoming metastable) and inducing explosive vaporization. It is a cheaper alternative due to the lower price of CW laser light source and, however, usually needs absorbing media in the liquid to be efficient. Recently, socalled plasmonic bubbles, named after the surface plasmonic effect (combined oscillation of electrons in metallic nanoparticles), are used to generate them, as enhanced 
Table 4 Overview of parameters for the pulsed laser-induced cavitation bubbles, from different sources

\begin{tabular}{|c|c|c|c|c|c|}
\hline Reference & $\begin{array}{l}\text { Laser type and wave- } \\
\text { length }\end{array}$ & Focusing optics & $\begin{array}{l}\text { Energy per pulse } \\
{[\mathrm{mJ} / \text { pulse]/duration }} \\
{[\mathrm{ns}]}\end{array}$ & $\begin{array}{l}\text { Bubble radius }[\mu \mathrm{m}] / \\
\text { lifetime }[\mu \mathrm{s}]\end{array}$ & $\begin{array}{l}\text { Bubble energy, } \\
\text { first oscillation } \\
{[\mu \mathrm{J}]}\end{array}$ \\
\hline Sato et al. (2013) & $\begin{array}{l}\text { Nd:YAG Q-swiched } \\
\quad(532 \mathrm{~nm})\end{array}$ & - & $3.2-10.6 / 5$ & $1000 / 220^{*}$ & $420^{*}$ \\
\hline Hellman et al. (2007) & $\begin{array}{l}\text { Nd:YAG Q-swiched } \\
\quad(532 \mathrm{~nm})\end{array}$ & $\begin{array}{l}\text { 40x, NA } 0.8 \text { or } 20 \times \text { NA } \\
0.5 \text { objective }\end{array}$ & $20-25 / 6$ & $230 \times 110 \times 50 / 50$ & $0.53^{*}$ \\
\hline Quinto-Su et al. (2008) & $\begin{array}{l}\text { Nd:YAG Q-swiched } \\
(532 \mathrm{~nm})\end{array}$ & $20 \times$ NA 0.75 Objective & $0.26-0.335 / 6$ & $20-50 / 5$ & $0.0033-0.052^{*}$ \\
\hline Vogel et al. (1994) & $\begin{array}{l}\text { Nd:YAG Q-swiched } \\
\quad(1064 \mathrm{~nm})\end{array}$ & $\begin{array}{l}\text { Ophtalmic lens } \\
\text { Rodenstock RAK NA } \\
0.41-0.47\end{array}$ & $0.05-20 / 0.03$ and 6 & $0.2-2.2 / 18-201$ & $3.3-5000$ \\
\hline Vogel et al. (1996) & $\begin{array}{l}\text { Nd:YAG Q-swiched } \\
\quad(1064 \mathrm{~nm})\end{array}$ & $\begin{array}{l}\text { Ophtalmic lens } \\
\text { Rodenstock RYK NA } \\
0.32-0.50\end{array}$ & $0.05-10 / 0.03$ and 6 & $0.225-1.82 / 20-167$ & $4.7-2500$ \\
\hline Sinibaldi et al. (2019) & $\begin{array}{l}\text { Nd:YAG Q-swiched } \\
\quad(532 \mathrm{~nm})\end{array}$ & $\begin{array}{l}\text { Parabolic mirror } \\
\mathrm{f}=54,45 \mathrm{~mm}, \mathrm{NA} \\
0.2-0.8\end{array}$ & $5-25 / 8$ & $1300-2000 / 240-380$ & $500-3500 *$ \\
\hline Zhang et al. (2019) & $\begin{array}{l}\text { Nd:YAG Q-swiched } \\
(532 \mathrm{~nm})\end{array}$ & - & $0.115 / 8$ & $620-1140 / 80-110$ & $89-550 *$ \\
\hline Tinguely et al. (2012) & $\begin{array}{l}\text { Nd:YAG Q-swiched } \\
\quad(532 \mathrm{~nm})\end{array}$ & $\begin{array}{l}\text { Parabolic mirror } \\
\mathrm{f}=54.5 \mathrm{~mm}, \mathrm{NA} 0.8\end{array}$ & $55-230 / 8$ & $\begin{array}{l}\text { 2000-5600/1000-3500 } \\
\text { (varying amb. pressure) }\end{array}$ & $2000-11,000$ \\
\hline Ohl (2002b) & $\begin{array}{l}\text { Nd:YAG Q-swiched } \\
\quad(1064 \mathrm{~nm})\end{array}$ & - & $10-30 / 8$ & $600-1800 />50$ & $90-2440 *$ \\
\hline Li et al. (2017) & $\begin{array}{l}\text { Nd:YAG Q-swiched } \\
\quad(532 \mathrm{~nm})\end{array}$ & $\begin{array}{l}63 \times \text { objective } \\
\text { (ZEISS LD plan neo- } \\
\text { fluar) }\end{array}$ & $\sim 0.01 / 5$ & $\begin{array}{l}50 / \sim 4 \text { (confined } \\
\text { volume) }\end{array}$ & $0.052 *$ \\
\hline Zwaan et al. (2007) & $\begin{array}{l}\text { Nd:YAG Q-swiched } \\
\quad(532 \mathrm{~nm})\end{array}$ & $\begin{array}{l}40 \times \text { objective } \\
(\mathrm{CF} 40 \text { Carl ZEISS) }\end{array}$ & $5-50 / 6$ & $43 / 7$ & $0.033^{*}$ \\
\hline Dijkink and Ohl (2008) & $\begin{array}{l}\text { Nd:YAG Q-swiched } \\
(532 \mathrm{~nm})\end{array}$ & $\begin{array}{l}10 \times \text { NA } 0.25 \\
\text { objective }\end{array}$ & $10-50 / 6$ & $50 / \sim 10$ & $0.052 *$ \\
\hline Quinto-Su et al. (2009) & $\begin{array}{l}\text { Nd:YAG Q-swiched } \\
(532 \mathrm{~nm})\end{array}$ & $\begin{array}{l}20 \times \text { NA } 0.7 \\
\text { Objective }\end{array}$ & $-/ 6$ & $60 / 11-18$ & $0.09 *$ \\
\hline Akhatov et al. (2001) & $\begin{array}{l}\text { Nd:YAG Q-swiched } \\
(1064 \mathrm{~nm})\end{array}$ & - & $20 / 8$ & $500-3000 / \sim 100$ & $52.3-11.3^{*}$ \\
\hline Brujan (2008) & $\begin{array}{l}\text { Nd:YAG Q-swiched } \\
\quad(1064 \mathrm{~nm})\end{array}$ & Lens NA $0.48^{*}$ & $-/ 6$ & $400 / \sim 80$ & 26.8 \\
\hline Oguri and Ando (2018) & $\begin{array}{l}\text { Nd:YAG Q-swiched } \\
(532 \text { and } 1064 \mathrm{~nm})\end{array}$ & $40 \times$ NA 0.6 objective & $1.4 / 6$ & - & - \\
\hline $\begin{array}{l}\text { Brujan and Vogel } \\
\text { (2006) }\end{array}$ & $\begin{array}{l}\text { Nd:YAG Q-swiched } \\
(1064 \mathrm{~nm})\end{array}$ & Lens NA $0.7 *$ & $1-10 / 6$ & $1700 / 300$ & $26.7-2057^{*}$ \\
\hline Quinto-Su et al. (2014) & $\begin{array}{l}\text { Nd:YAG Q-swiched } \\
\quad(532 \mathrm{~nm})\end{array}$ & $\begin{array}{l}40 \times \text { NA } 0.8 \text { water } \\
\text { immersion objective }\end{array}$ & $0.001-0.009 / 6$ & $10-35 / 10$ & $0.00,042-0.018^{*}$ \\
\hline
\end{tabular}

Data marked with asterisk $(*)$ are not provided by the authors of the reference, but are approximated from the corresponding article for comparison

absorption of visible light can be achieved. For this reason, it has become a popular technique to study nucleation mechanism and growth dynamics of vapor bubbles (Wang et al. 2017, 2018; Zaytsev et al. 2020). Using CW lasers, nanoparticles can be heated up quickly (ns- $\mu$ s range) and to high temperatures, causing explosive growth of photothermal bubbles. Similarly, an optical fiber can be used to generate bubbles at the fiber-liquid interphase, as intense laser light is absorbed and rapidly heats up the liquid.
Depending on the light source, the bubble generation at the fiber tip can be either thermal (CW with absorptive coating tip) or stress confinement (short pulsed lasers), with studies often linked to medical procedures (Mohammadzadeh et al. 2016). However, as all these methods have so far been limited to surface or near surface bubble generation, they are not suitable for generating spherical bubbles in bulk liquid, and are therefore not included in the scope of this study. 


\subsection{Rayleigh-Plesset model}

The Rayleigh-Plesset equation (RPE) is derived from first principles and is often used to model the bubble dynamics in idealized cases (Leighton 2007):

$\frac{p_{v}(t)-p_{\infty}(t)}{\rho_{l}}=R \frac{d^{2} R}{d t^{2}}+\frac{3}{2}\left(\frac{d R}{d t}\right)^{2}+\frac{4 v_{l}}{R} \frac{d R}{d t}+\frac{2 \gamma}{\rho_{l} R}$,

where the left side term defines the pressure conditions in the bubble $\left(\mathrm{p}_{\mathrm{b}}\right)$ and the surrounding infinite liquid $\left(\mathrm{p}_{\infty}\right)$. The first and the second right side terms give the inertia of the bubble, the third term defines the viscosity $\left(v_{1}\right)$ and the fourth defines the surface tension effects $(\gamma)$. The model is based on several assumptions (Prosperetti 1982): The bubble interphase is perfectly spherical throughout the growth/ collapse, with homogeneous bubble content, and no body forces acting on bubble during its lifetime. The liquid surrounding the bubble is infinite and considered incompressible, with isothermal conditions applying. The driving force acts on the interface of the bubble, with bulk liquid viscosity and interfacial tension assumed constant. It is derived from Bernoulli's theorem/conservation laws and was originally developed to describe the collapse of an empty cavity in an infinite incompressible liquid. This initial simplification is the so-called Rayleigh collapse model, where the bubble collapses under the instantaneous pressure change in the liquid. The Rayleigh collapse model simplifications allow us to approximate the bubble collapse time $\left(\tau_{\mathrm{c}}\right)$ from its maximum size and the ambient pressure, via the Rayleigh collapse time equation (Brennen 1995):

$\tau_{c}=0.915 * R_{\max } \sqrt{\rho_{l} /\left(p_{0}-p_{v}\right)}$,

where the $R_{\max }$ is the maximum bubble radius, $\rho_{1}$ is the density of the liquid and the liquid ambient $\left(\mathrm{p}_{0}\right)$ and vapor pressure $\left(\mathrm{p}_{\mathrm{v}}\right)$.

Although there are many assumptions to the RPE, it has been often times demonstrated to adequately model bubble dynamics in experimental and real-world settings. There are more elaborate models developed for specialized applications, but the simplification allows it to be a robust model for dynamics of a spherical vapor bubble collapse. As the RPE has no closed-form solution, it is often tackled numerically. However, there have also been several analytical solutions proposed (Kudryashov and Sinelshchikov 2014, 2015; Mancas and Rosu 2016); for our purpose, we used an accurate analytical solution for the bubble dynamics, offered by (Obreschkow et al. 2012).

\section{Experimental setup}

We used four different vapor bubble generation techniques, each producing bubbles by a specific mechanism. The TAM, HVD and LVD methods were modified compared to previous experimental setups, while the pulsed laser-induced techniques is used as a benchmark technique and as such remains unmodified. Compared to the previous spring powered design (Dular and Coutier-Delgosha 2013), the TAM method uses pneumatic propulsion with a quick release mechanism, which has shown much better tunability and repeatability of the produced bubbles. Furthermore, the bubble nucleation system has been modified, using a thin needle attached to the bottom of the moving tube. This helps to maintain bubble sphericity, as there is no relative motion between the nucleation site and the liquid. Several tube end fittings were tested in order to tackle the unwanted nucleation on the tube walls, which perturb the natural bubbles dynamics. This has extended the usable range of the device, as it has been one of the most problematic issues with the technique. An accelerometer was used to determine the deceleration encountered at impact. The LVD was based on the design of (Goh et al. 2013), but modified to allow for selectable capacitance in addition to varying voltage. This allows for greater control of the energy delivered in a single discharge. Lastly, the piezoelectric HVD technique was used with tungsten electrodes, allowing bubbles to be discharged in bulk liquid, as opposed to previously reported experimental setups (Avila et al. 2015), which were limited to hemispherical (at wall) bubbles. The implementation of the electrodes also allowed for the bubble size to be controlled, via the interelectrode gap, giving the HVD technique an element of tunability it lacked beforehand. Although a more powerful piezoelectric sparker was used, the method remains very much user safe, due to the low energy involved in a single discharge, as opposed to the capacitor based HVD circuits.

The liquid (except for the tube arrest method) was placed in a rectangular glass container, $90 \times 90 \times 90 \mathrm{~mm}$ in size as shown in Fig. 3, filled to about $2 / 3$ height with distilled water, unless otherwise specified. For the electrical discharge techniques, the electrodes could be positioned in $\mathrm{x}, \mathrm{y}$ and $\mathrm{z}$ directions with a micropositioning system and were electrically isolated throughout. High-speed images were recorded of the resulting bubble dynamics with the Photron Fastcam SA-Z 2100 K-M-64 GB and the AS-F VR Micro-Nikkor $105 \mathrm{~mm}$ camera objective, with backlight illumination provided by a LED. For measurements of the LVD 95,000 or 150000FPS were used, while recordings for HVD and laser discharges 400000FPS always using $250 \mathrm{~ns}$ shutter speed. For laser-induced breakdown, the 
Fig. 3 Laser a and LVD/HVD b induced bubble experimental liquid container, lens, electrode positioning and image acquisition. Laser-induced bubbles are produced by the focused laser light, while for the two electrical discharge methods, electrodes are used. The LVD copper electrodes are in contact, while the HVD tungsten electrodes have a small gap in between. Backlight illumination is used for the image acquisition

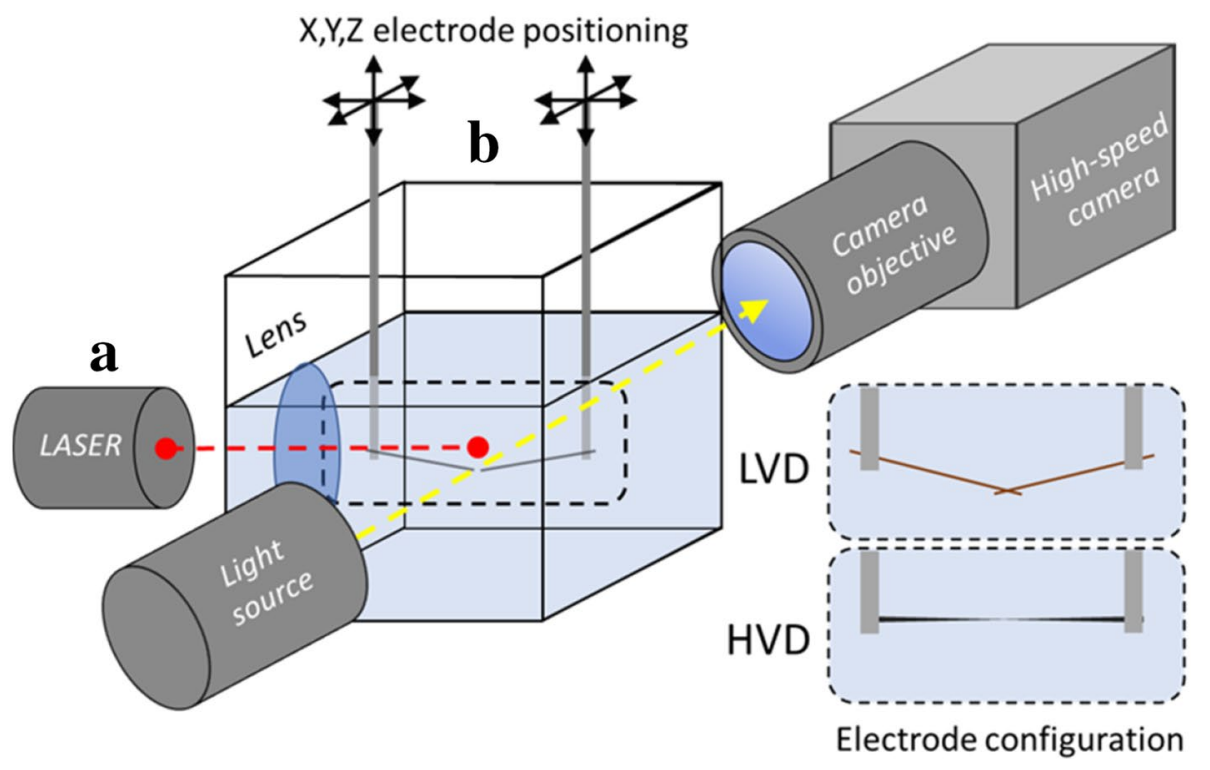

same shutter speed was used, only at 210,000 or 480,000 FPS, while for acquisition with the tube arrest method was at 100000FPS and $1 \mu$ s shutter. Distilled water was used as the working liquid, at $25{ }^{\circ} \mathrm{C}$ and ambient pressure $\mathrm{p}_{0}$. Degassing was performed for the tube arrest method, as it prevents or minimizes nucleation on the vessel walls. For experiments with varying electrical conductivity, $\mathrm{NaCl}$ was added to distilled water. Conductivity was measured with the HQ430D Multi-Parameter Meter from HACH. Temperature was monitored with a K-type thermocouple.

\subsection{High-voltage discharge}

A simple piezoelectric igniter (CBS-PZ-G158 $16 \mathrm{kV}-\mathrm{B} 4$ from Conrad electronic SE) was used to produce a $16 \mathrm{kV}$ pulsed spark discharge in the low-conductivity distilled water. A spring-loaded mechanical design ensures a repeatable strike on the piezoelectric ceramic actuator. This setup is a considerably cheaper alternative to the costly high-voltage supply and circuitry typically needed for high-voltage discharge studies (Avila et al. 2015). The low-energy piezoelectric discharge can simplify the setup by providing a pulsed high-voltage source, with the disadvantage of the loss of voltage control. The capacitance of the piezosparker was estimated at roughly $30 \mathrm{pF}$. Tungsten carbide needles were used for the electrodes, as they can withstand the high temperatures generated at the spark discharge and limit the erosion of the electrode material. The electrodes used are tungsten needles RS-6065 from Roboz surgical instrument co., with a $500 \mu \mathrm{m}$ stem, tapering off to a micron-sized sharp tip. The electrodes were coated with nail polish, except at the conical tip where they are in contact with the bubbles.

\subsection{Low-voltage discharge}

A setup, similar to the one used in (Goh et al. 2013), was modified to allow a selectable capacitance of the electrical circuit (Fig. 4). It contains a transistor, which controls the charging and discharging of the capacitor bank. One 6800 $\mu \mathrm{F}$ and three $2200 \mu \mathrm{F}$ capacitors $( \pm 20 \%)$ can be individually engaged by mechanical switches, offering 7 different capacity settings. The sparking circuitry allows for a rapid release of the stored energy in the capacitors to the electrodes. As the electrodes are in contact, the smallest cross section in the circuit is the contact point. The MOSFET (IXFH75N10 from IXYS semiconductors) releases the current in roughly $100 \mathrm{~ns}$, a bubble appears due to Joule heating at this point. The discharge lasts between 0.3 and $0.8 \mathrm{~ms}$, depending on the voltage and capacitance settings. A 2231 A-30-3 power supply from Keithley was used for the charging voltage between 20 and $60 \mathrm{~V}$. A typical $155 \mu \mathrm{m}$ copper wire was used for the electrodes with the tips crossed over for contact.

\subsection{Pulsed laser method}

A similar setup was used as in (Horvat et al. 2018) and was used for the pulsed laser experiment, shown in Fig. 5. A liquid container (a) with the wall integrated focusing lens (b) with the numerical aperture value of 0.23 was used, which helps minimize losses in the optical system. The beam expander (b), splitter (c) and attenuator condition the laser beam from the $1064 \mathrm{~nm}$ Q-switched Nd:YAG pulsed laser source (e), which has a pulse duration in ns range and up to $15 \mathrm{~mJ}$ with minimum attenuation. Laser pulses energy is measured at (f), assuring the variance is kept below $1.5 \%$. A trigger photodiode $(\mathrm{g})$ is used to synchronize the laser pulse and the high-speed camera (i). 


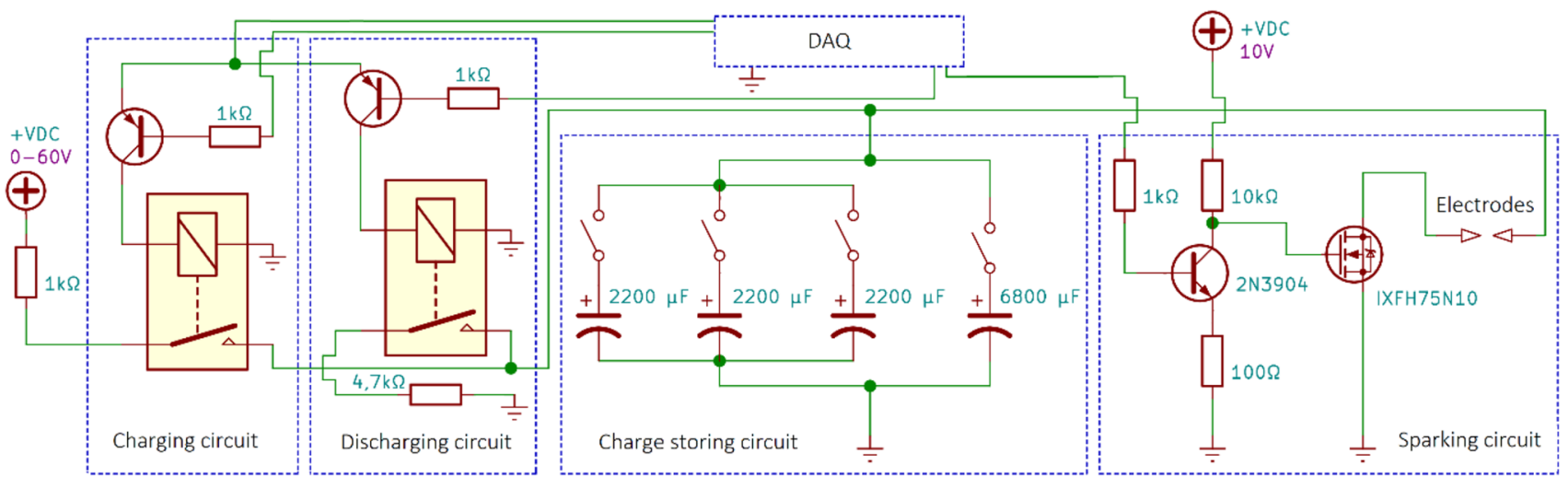

Fig. 4 Low-voltage discharge electrical circuitry, based on experimental setup in (Goh et al. 2013), modified with 4 capacitor options for variable capacitance

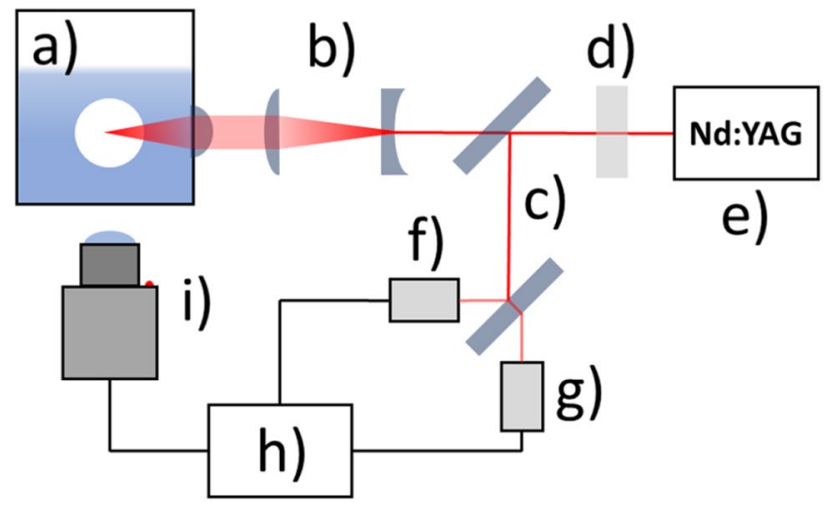

Fig. 5 Pulsed laser experimental setup and its components: a liquid container, $\mathbf{b}$ beam expander and focusing optics, $\mathbf{c}$ beam splitters, $\mathbf{d}$ attenuator, e Q-swiched $1064 \mathrm{~nm}$ Nd:YAG laser, f energy meter, $\mathbf{g}$ trigger photodiode, $\mathbf{h}$ computer, $\mathbf{i}$ high-speed camera. A similar setup was used as in (Horvat et al. 2018)

\subsection{Tube arrest method}

Several modifications were implemented in our tube arrest setup, compared to traditional configurations. Firstly, we used compressed air to power the tube into motion, as opposed to the spring powered devices. Pneumatic cylinders can provide a controllable impact speed without a significant bounce back seen with the spring-loaded devices. As most modern laboratories have a compressed air source, this does not present a huge cost increase for the setup. Furthermore, the travel length can be adjusted, so the velocity of the tube just before impact can be monitored by the driving pressure or the travel distance. The nucleation point was also addressed, as it is a key feature for bubble dynamics. In our setup, a shorter nucleation rod was mounted at the bottom of the tube, so the liquid and wall velocity are equal. While this has the disadvantage of hindering observation, it can help with the bubble shape, which otherwise tend to become ellipsoid in typical top suspended nucleation rods. An acrylic glass tube of $50 \mathrm{~mm}$ outer and $40 \mathrm{~mm}$ inner diameter was used for the liquid container, as it provides an optimal balance between rigidity, transparency and impact strength for the liquid vessel.

At the top of the device, a threaded stop cap can be adjusted and locked into position by tightening the nut, thereby fixing the tube travel distance (Fig. 6(A)). The tube guides (B) maintain the tube (C) on a straight trajectory during movement. Acrylic glass was found to be the most suitable material for a tube this size, while glass is too brittle and polycarbonate to flexible. As the triggering mechanism releases (D) the force, stored in the two pneumatic cylinders $(E)(\varphi=50 \mathrm{~mm})$ under the set pressure, the tube is propelled toward the stop cap and brought to an abrupt stop. From this point onwards, the mechanism, previously described in Fig. 2, takes over and drives the bubble generation with an ab initio tension wave. Many options were tested for the tube end $(\mathrm{F})$, such as glass borosilicate bottles and acrylic plugs; however, the best results were shown for a rigid rubber cork (F-1) with the hypodermic needle (F-2), coated by polyurethane coating $(\mathrm{F}-3)$ to prevent nucleation on surface of the rubber. The stainless steel needle was 20 G $0.90 \times 70 \mathrm{~mm}$, containing a smaller $30 \mathrm{G} 0.3 \times 12 \mathrm{~mm}$ needle, and a syringe valve (F-4) was placed below, allowing us to reform the nucleation bubble after each event, while stopping the gas inflow during bubble growth. An air bubble was pushed through the needle before each recording. An acrylic glass container filled with water was used to submerge the tube end in order to minimize optical aberrations, when imaging through the curved tube wall. The tube was filled to $70 \mathrm{~cm}$ with distilled water, before being degassed for at least $10 \mathrm{~min}$, with a vacuum pump. The tube travel was 3 to $4 \mathrm{~mm}$, which showed better results, compared to long travel distances. A Brüel and Kjær 2635 charge amplifier, 


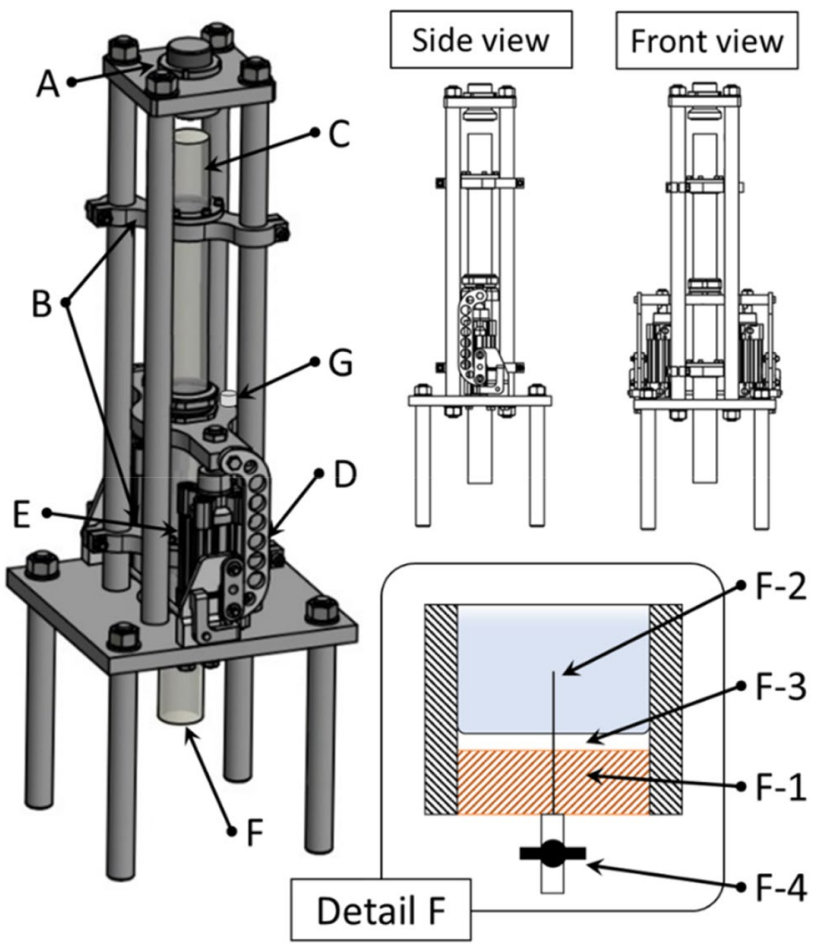

Fig. 6 Tube arrest experimental setup with listed components: a the adjustable stop cap with nut, $\mathbf{b}$ metallic bracket with Teflon tube guides, c acrylic tube, $\mathbf{d}$ mechanical triggering mechanism, e pneumatic cylinders (one on each side), $\mathbf{f}$ tube end: F-1) rubber cork, F-2) nucleation needle, F-3) polyurethane coating, F-4) valve, g accelerometer

along with the 4387 accelerometer (G) mounted on the tube housing, was used to monitor the impact deceleration. A 1.5-1 reservoir for compressed air is mounted before the cylinders, to provide ample gas supply close to the cylinder inlets (omitted in figure for clarity).

\section{Results}

The four commonly used methods for producing a single transient spherical vapor bubble in bulk liquid are shown in Fig. 7a-d, where we can see the bubble growth, collapse and the first rebound for all four bubble methods discussed. The initial conditions $\left(\mathrm{R}_{0}\right)$ are displayed, along with the bubbles shown at half $\left(\mathrm{R}_{1 / 2}\right)$, full $\left(\mathrm{R}_{\max }\right)$ and collapse radii $\left(\mathrm{R}_{\mathrm{c}}\right)$. All methods are capable of producing spherical bubbles throughout the first oscillation, despite the presence of electrodes and the nucleation needle. The growth $\left(\tau_{\mathrm{g}}\right)$ and the collapse $\left(\tau_{c}\right)$ periods present the bubble lifetime of the first oscillation $(\tau)$. For all but the laser (d) technique, the following rebounds are distorted by the perturbation of the solid surfaces $\left(\mathrm{R}_{\max , 2}\right)$. The electrical discharge methods can often form two rebound bubbles, due to the bubble breakup and jetting in the direction of the electrodes, shown in Fig. $7 \mathrm{f}$ at maximum rebound radius. The methods compared herein are driven by different physical principles, as covered in the theoretical background chapter, and are thus governed by parameters specific to each method. Figure 8 shows the bubble maximum volume $\left(\mathrm{V}_{\max }\right)$ produced by the tube arrest method at different impact deceleration values. The four points are at $0.5,1,1.5$ and 2 bar driving pressure applied to the two pneumatic cylinders. Nucleation was limited to the needle point for all cases except the last point, where occasionally a second bubble would appear at the point where the needle enters the tube. Bubble growth was not observed for values below $1000 \mathrm{~m} / \mathrm{s}^{2}$, for the current experimental setup. At higher decelerations, the bubble tends to be deformed by surface instabilities and has been observed to produce jets from the nucleation tube, deforming the bubble shape as seen in Fig. 7e. Bubbles show extended growth times at lower and moderate deceleration values, as the $\mathrm{R}_{\max }$ occurs at roughly $2 / 3$ of the bubble lifetime, shown in Fig. $7 \mathrm{a}$. At the higher range, the normalized growth time is shortened, perhaps due to the accompanying bubble interference. An isochronous case is difficult to achieve with this method, due to the recoiled initial tension wave affecting the bubble, throughout its lifetime.

Figure 9a shows the variable parameters determining the LVD bubble maximum size. For LVD, the voltage and capacitance were varied, at fixed $4400 \mu \mathrm{F}$ capacitance for the former and $40 \mathrm{~V}$ voltage supply for the latter. This is in accordance with the energy stored in the capacitor, which increases linearly with capacitance and follows the square root trend for increasing voltage. From Fig. 7b, we can see the growth time is also elongated for the LVD, owing to the extended spark discharge, which cannot be considered pulsed in respect to the bubble lifetime, as the discharge can be seen at half, and even maximum bubble radius. On the other hand, for HVD the initial energy pulse, as shown in Fig. $7 \mathrm{c}$ at $\mathrm{R}_{0}$, will drive the bubble growth and collapse. For $\mathrm{HVD}$, the electrode gap was gradually increased from 50 to $500 \mu \mathrm{m}$ in $50 \mu \mathrm{m}$ increments. Figure $9 \mathrm{~b}$ shows the useful range between 50 and $300 \mu \mathrm{m}$, while discharge can occur up to $450 \mu \mathrm{m}$ with less than $100 \%$ sparking success rate. HVD shows the normalized growth times $\left(\tau_{\mathrm{g}} / \tau\right)$ slightly elongated at shorter electrode gaps, leveling out at isochronous growth and collapse periods up to $300 \mu \mathrm{m}$ electrode gap and stays level for the rest of the range.

We also tested the suitability of the HVD and LVD techniques in el. conductive water, as is can be important for various fields, for example studying erosion in tap/salt water or studies in biological tissues interactions, which require isotonic conditions to be representable. Water electrolytic conductivity effect on bubble size is shown in Fig. 9c. For LVD bubbles, we used $4400 \mu \mathrm{F}$ capacitance and $40 \mathrm{~V}$ for bubble generation, while the HVD interelectrode gap was 

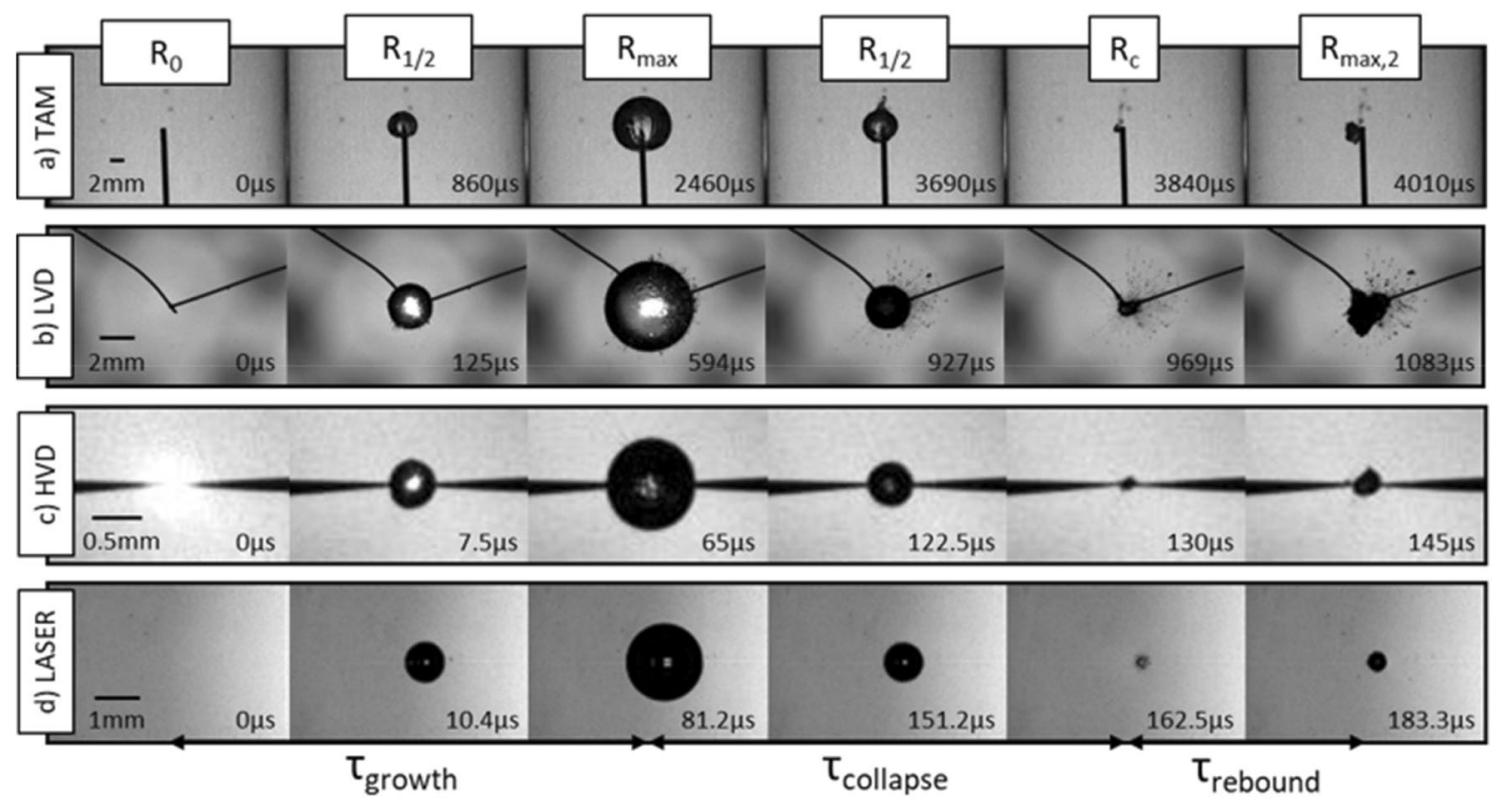
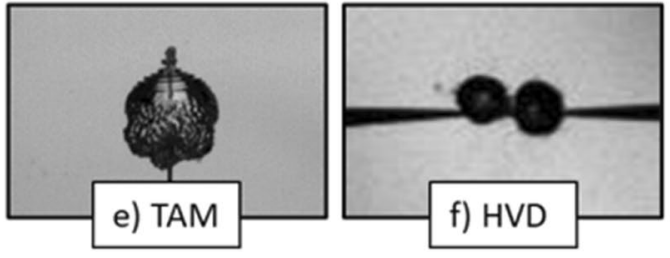

Fig. 7 Typical bubble dynamics of the four different methods: a tube arrest method (TAM) bubble, b low- (LVD) and $\mathbf{c}$ high-voltage discharge (HVD) bubble, d laser-induced bubble. Bubble series a through $\mathbf{d}$ display the layout before vapor generation $\left(\mathrm{R}_{0}\right)$, the bubbles at half maximum $\left(\mathrm{R}_{1 / 2}\right)$ and maximum size $\left(\mathrm{R}_{\max } ; \mathrm{TAM}=4.34 \mathrm{~mm}\right.$, $\mathrm{LVD}=2.6 \mathrm{~mm}, \mathrm{HVD}=0.66 \mathrm{~mm}, \mathrm{LASER}=0.86 \mathrm{~mm}$ ), at collapse
$\left(\mathrm{R}_{\mathrm{c}}\right)$, and at the maximum rebound radius $\left(\mathrm{R}_{\max , 2}\right)$. e Surface instabilities and jets that can occur with TAM. f Double bubble rebound for HVD. g Electrode breakup and the non-spherical bubble collapse often seen with LVD. h Laser-induced bubble in tap water with small bubbles nucleating on impurities, driven by the initial shockwave
Fig. $8 \mathrm{~V}_{\max }$-bubble maximum volume (right) and the corresponding $\tau_{\mathrm{g}} / \tau_{\mathrm{TAM}}$-normalized growth times (left) at different impact decelerations, generated with TAM - tube arrest method. The dashed line presents the idealized isochronous growth and collapse period (RPE case)

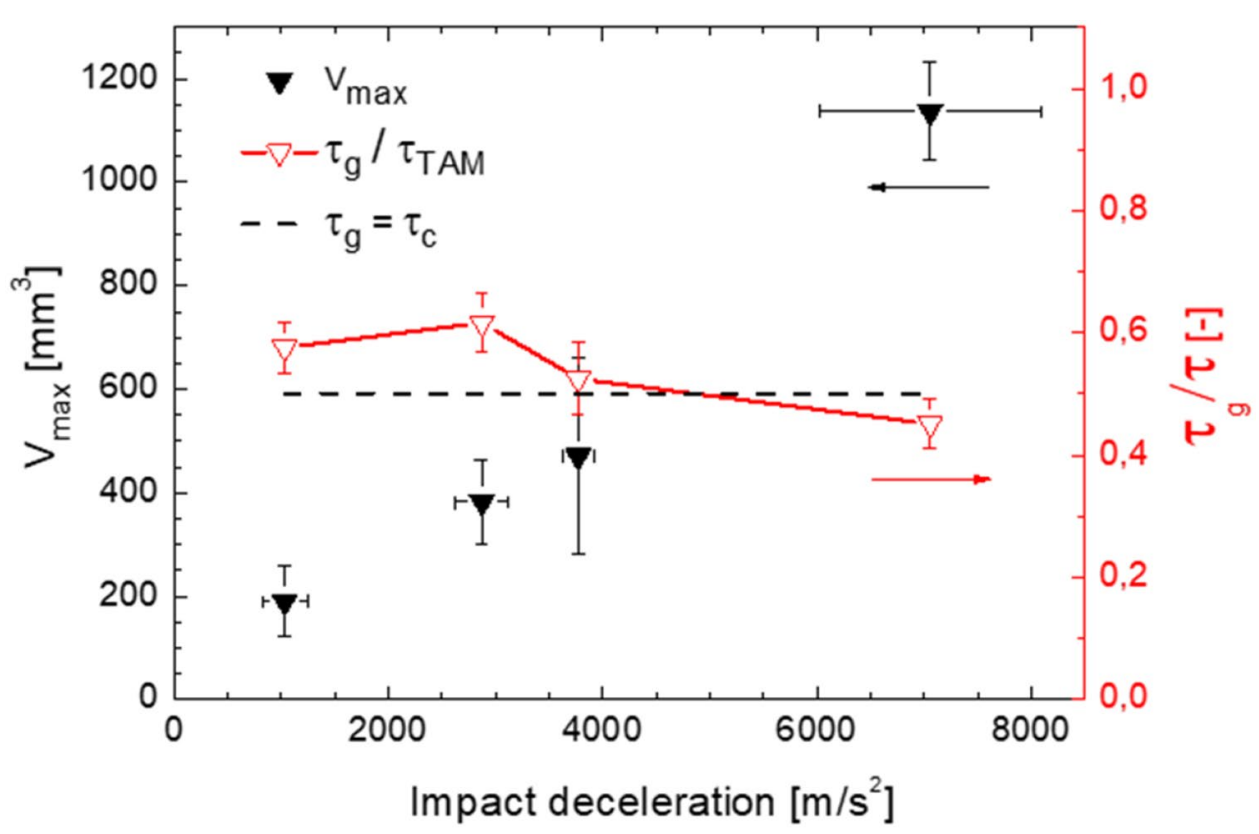



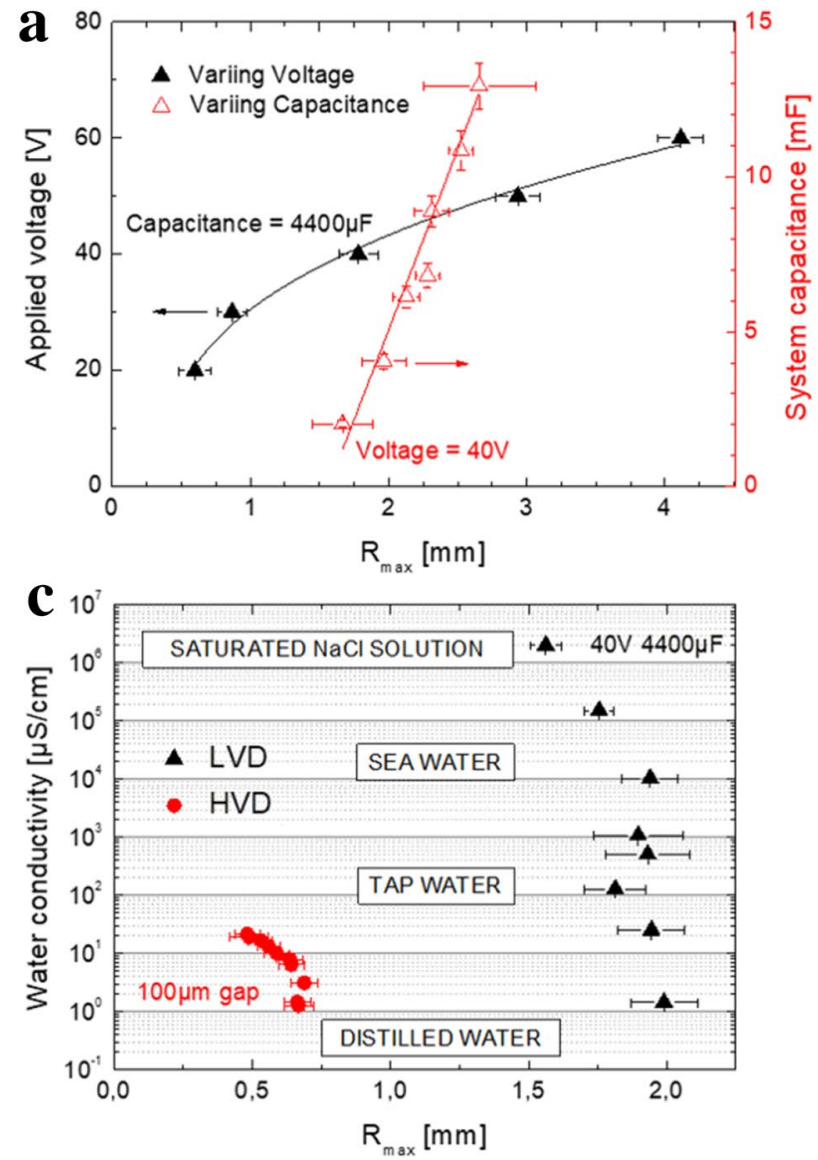

Fig. 9 a LVD bubble size with varying voltage (left $Y$ axis) and capacitance (right $\mathrm{Y}$ axis), b HVD bubble volume (left $\mathrm{Y}$ axis) and successful bubble production percentage as well as the normalized bubble growth time (right $\mathrm{Y}$ axis) with varying interelectrode distance, c LVD and HVD bubble size at different water electrical conductivity, $\mathbf{d}$ LVD and HVD bubble size (left $\mathrm{Y}$ axis) and the $\tau_{\mathrm{g}} / \tau-$

$100 \mu \mathrm{m}$, both at $25^{\circ} \mathrm{C}$. Electrolytic conductivity seems to play a critical role with the HVD, as with the current setup it only generates bubbles below $20 \mu \mathrm{S} / \mathrm{cm}$ water conductivity. An electrically non-conductive $0.8 \mathrm{M}$ saccharose solution was tested and successfully produced a discharge, making it a viable option for use with biological samples (preventing osmotic shock). The LVD is much less affected by the el. conductivity of the solution, as the electrodes are in contact, and the energy is dissipated to the liquid by joule heating. This makes it useful practically throughout the conductivity range of saline solution, from distilled water to saturated $\mathrm{NaCl}$ solution; however, the frequent electrode disintegration has to be considered as samples might get contaminated by the electrode fragments (Fig. $7 \mathrm{~g}$ ). Little influence on the bubble size was observed down to $10 \mathrm{mS} / \mathrm{cm}$ and after that a bubble radius decline was observed, while maintaining discharge capability throughout the range. Figure $9 \mathrm{~d}$ shows the temperature influence on bubble size and its growth period
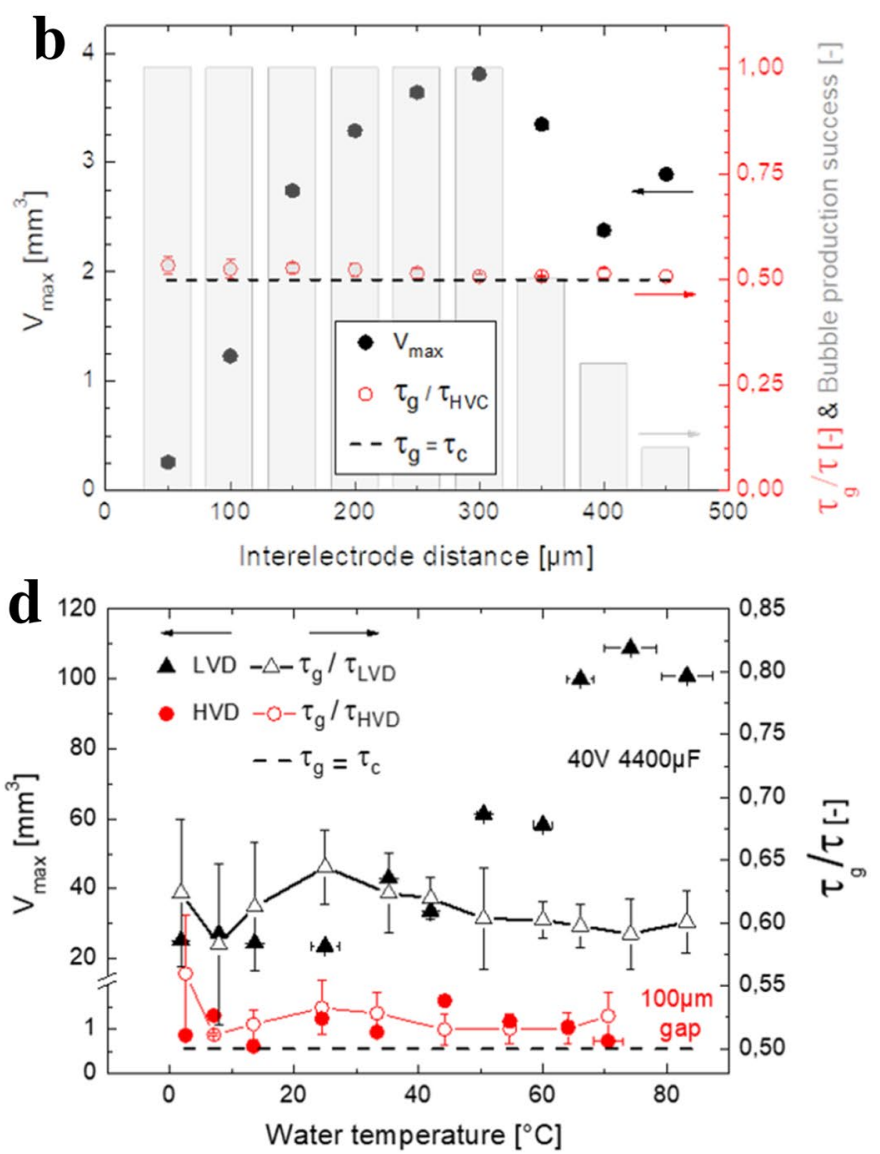

normalized growth time (right $\mathrm{Y}$ axis-growth/lifetime ratio) with varying water temperature. Each point is averaged over 5 (LVD) or 10 (HVD) bubble measurement events. For $\mathbf{b}$ and $\mathbf{d}$, the dashed line shows the ideal bubble growth time from the Rayleigh-Plesset equation, with isochronous $\tau_{\mathrm{g}}$ growth and $\tau_{\mathrm{c}}$ collapse times

in relation to the bubble lifetime. Firstly, we can see that for the LVD the bubble volume is, as expected, increasing with rising liquid temperature, due to increasing vapor pressure facilitating the phase transition. Surprisingly, this is not the case for HVD, where the bubble does not show a rising trend. Furthermore, we can see that the normalized growth times $\left(\tau_{\mathrm{g}} / \tau\right)$ for both techniques are similar in trend, but at very different values. LVD produces much longer $\left(\tau_{\mathrm{g}} / \tau \approx\right.$ $0.6-0.65)$ growth periods compared to $\operatorname{HVD}\left(\tau_{\mathrm{g}} / \tau \approx 0.5\right)$, where the latter are very close to the idealized Rayleigh model $\left(\tau_{\mathrm{g}} / \tau=0.5\right)$. Both peak at low and ambient temperatures and do not show a significant rise with the increasing liquid temperatures. Moreover, the peak bubble volumes seem inversely proportional the normalized growth times.

In Fig. 10, we see that for the reference laser-based technique, a constant $30 \%$ fraction of available laser pulse energy $\left(\mathrm{E}_{\mathrm{L}}\right)$ is assumed to be deposited in the induced bubble. However, the observed relation shows that the radius grows 


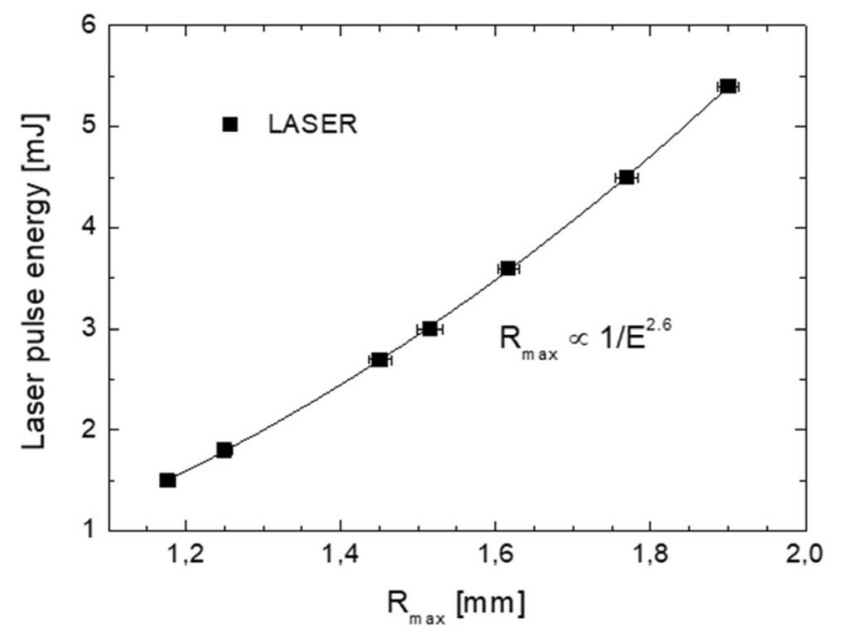

Fig. 10 Laser-induced bubble radius at different laser pulse energies

slightly faster with relation $R_{\max } \propto E_{L}^{1 / 2.6}$. This implies that a slightly larger fraction of laser pulse energy is deposited in the bubble with increasing pulse energy-a result consistent with observations in (Vogel et al. 1999). Bubble lifetimes follow the RPE curve $\left(\tau_{\text {LASER }}=0.5\right)$; therefore, the data are omitted from graphs for clarity. Water electrical conductivity as a parameter for laser-induced bubble generation was not studied, because it is not expected to have any influence. However, water purity effects were briefly analyzed for tap water, distilled water and ultrapure water $(\mathrm{R}>18$ $\mathrm{M} \Omega / \mathrm{cm}$ ). Difference in bubble radius was not observed due to insufficient image resolution, while difference in bubble lifetime is possible to observe at high framerate. Bubbles generated in ultrapure water had approximately $3 \mu$ s longer lifetime than bubbles in distilled water, which in turn have approximately $4 \mu$ s longer lifetime compared to bubbles in tap water. Additionally, when distilled or tap water is used, small bubbles are generated due to laser pulse absorption on impurities and expanded by the negative pressure shockwave (Fig. 7h). These bubbles are not present for experiments in ultrapure or distilled water (Fig. 7d). The bubble radius evolution in regards to the bubble lifetime for each method compared is presented in Fig. 11. For the TAM case (blue line), the bubble temporal evolution curve follows the RPE predicted shape with a somewhat extended growth phase. Furthermore, it displays the lifetime roughly 4.7 times the predicted lifetime for a bubble its size, which is twice the Rayleigh collapse time, defined in Sect. 2.3. This is not the case for the laser-induced and HVD methods, as they follow the RPE evolution for an empty void (within experimental error), while LVD again shows an obvious elongation of the growth cycle. On the same graph, we can observe the sphericity at each timestep, which for all methods follow reasonably well the spherical bubble trend for the first bubble oscillation. Bubble circularity or in our case sphericity (as bubble symmetry is assumed in the horizontal axis) is evaluated on the isoperimetric quotient, which is the ratio of the area $(\mathrm{A})$ and perimeter $(\mathrm{P}) ; 4 \pi \mathrm{A} / \mathrm{P}^{2}$, sometimes termed compactness measure of a shape. If the bubble is perfectly spherical the value amounts to 1 , anything below is increasingly aspherical. This allows us to roughly evaluate whether the bubbles follow one of the assumptions made in the RPE, which is the perfect sphericity, throughout its lifetime. For the rebounds, bubbles start to deform for all methods, with least perturbation seen on the laser-induced bubbles. Figure 12 shows the plot of the bubbles recorded series at $25^{\circ} \mathrm{C}$ and $1 \mathrm{~atm}$ conditions, for all four methods. We plotted the bubble maximum radii and their lifetimes, reported or approximated from the literature and from the results of the current study, for all before mentioned techniques. The data from our study encompass all the bubbles measured

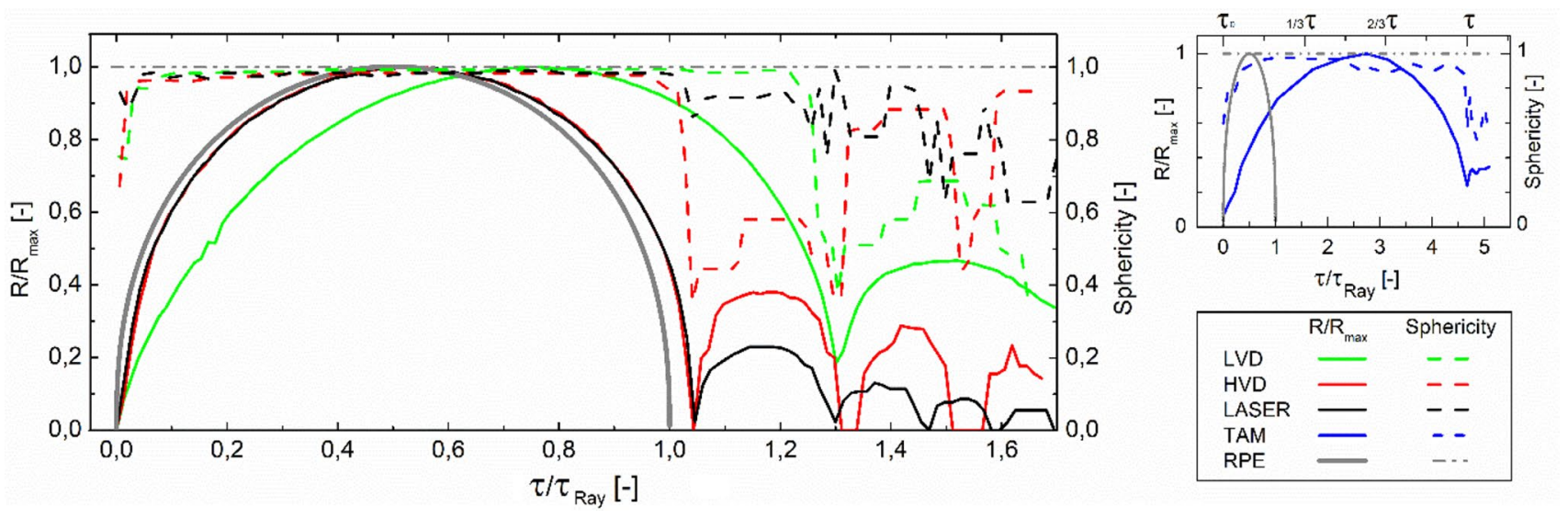

Fig. 11 Typical bubble dynamics for each method. The solid lines are the $R / R_{\max }$ values (left axis), and the dashed lines are the corresponding sphericity values (right axis) over time, normalized to the predicted Rayleigh lifetime for the particular bubble size $\left(\tau / \tau_{\text {Ray }}\right)$. The tube arrest method is shown in separate window, as the bubble lifetime $\left(\tau / \tau_{\text {Ray }}\right)$ is significantly longer compared to other methods 
Fig. 12 Bubble lifetime compared to bubble maximum radius, from the literature (full symbols) and current study (empty symbols). The dashed line presents the predicted lifetime of the bubbles at ambient pressure and temperature. The colored arrows represent the reported range for each technique

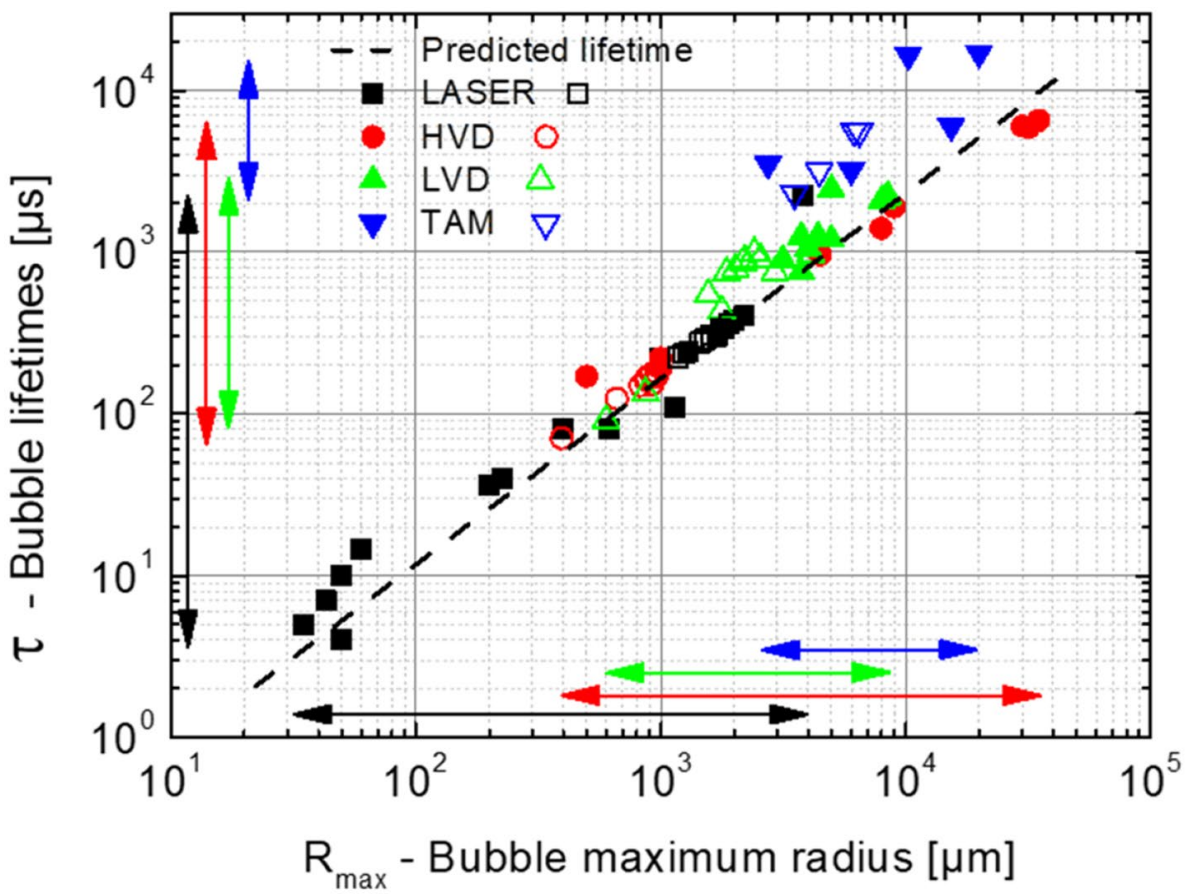

in different experiments for the various parameters (voltage, capacitance, electrode gap), in order to give an idea of the range of bubble size that can be expected from these techniques. Many different methods and experimental setups were used, so some discrepancies might occur due to approximations and/or inaccurate reporting from the literature. Expected bubble size and lifetime ranges are marked by the colored arrows corresponding to the technique, with the values tabulated in Tables 1, 2, 3 and 4 for each method group. The theoretically predicted lifetime $\left(\tau_{\text {Ray }}\right)$ is twice the Rayleigh collapse time and is marked with the dashed line. HVD and laser-induced bubbles tend to follow its trend, while LVD shows longer lifetimes than predicted, with the exception of the 20 and $30 \mathrm{~V}$ discharges (two empty green symbols on the dashed line). This discrepancy is even more prominent, for the TAM bubbles from the literature, as some at the higher range of the bubble size deviate considerably.

\section{Discussion}

\subsection{Tube arrest method}

Mechanical repeatability of the impact and liquid and surface quality are critical for repeatable results with TAM. In the current setup seems to have a usable range $1000-7000 \mathrm{~m} / \mathrm{s}^{2}$, with bubble control up to $3000 \mathrm{~m} / \mathrm{s}^{\mathrm{s}}$. From 3000 to $7000 \mathrm{~m} / \mathrm{s}^{\mathrm{s}}$, the surface instabilities and jetting from the needle can occur, perturbing the bubble dynamics, as shown in Fig. 7e. Beyond the usable range, accompanying nucleation can occur on the walls or in the liquid, perturbing the main bubble, while below this value the bubble formation is rarely observed. Thorough degassing and the use of hydrophilic surfaces in contact with water should extend the working range, as water would tend to nucleate less. However, by increasing the primary bubble even further, it could become limited by the tube walls. Therefore, the desired bubble radius range should be considered in the planning stage, so the appropriate tube diameter can be selected. For the TAM, the lifetimes are extended considerably compare to theory, due to the initial pressure wave recoils traveling up and down the tube liquid column, affecting the bubble dynamics and probably causing the instabilities which are often seen on the bubble surface (Fig. 7e). The surface instabilities can occur in the collapse phase of the bubble and which makes it difficult to determine the finite dynamics of the interphase, crucial for analysis of the jet formation. For a typical TAM bubble, several pressure wave passes would occur in its lifetime (3-5 ms), as every millisecond of bubble lifetime corresponds to the wave encountering the bubble approximately two times. For most of the range achieved with this technique, we can say that the growth phase presents up to $2 / 3$ of the bubble lifetime, while the for the largest bubbles we start to see collapse phase elongation (Fig. 8). The extended bubble lifetime, when comparing to the theoretically expected lifetime of a bubble its size $(\tau /$ $\tau_{\text {Ray }}$ ), suggests that the combined effect of the traveling pressure waves in the tube amount to an effective pressure the bubble experiences, significantly below ambient pressure, even though the tube is open to the environment. Prolonged 
lifetimes seem to be a common trend as shown in Fig. 12, as all reported TAM bubbles are above the predicted bubble Rayleigh lifetime. This also means the average velocity of the bubble interface is slower, which should be considered when studying bubble-structure interactions.

\subsection{Low- and high-voltage discharge}

LVD bubble size is governed by the stored energy in the capacitor $E_{c}=1 / 2 C V^{2}$, where $\mathrm{C}$ is the capacity of the circuit and $\mathrm{V}$ the supplied voltage, as shown in Fig. 9a. This seems to be the factor influencing bubble size, as the bubbles formed by increasing the capacitance of our storing circuit seem to increase linearly, while with increasing voltage, the bubble sizes follow the square root trend. Testing beyond $60 \mathrm{~V}$ was not undertaken, as it starts becoming dangerous for the user (safety one of the hallmarks of the technique) and is also the range of a typical benchtop power supply. For HVD, the main mechanism controlling bubble size was shown to be the interelectrode gap, as the voltage of the discharge cannot be moderated with our experimental setup. In Fig. 9b, we show a useful range between 50 and $300 \mu \mathrm{m}$, with discharges beyond this point becoming increasingly less likely. The bubble growth time can be assumed equal to the collapse time throughout the electrode gap range, meaning the bubbles follow the RPE bubble dynamics.

A notable limitation for HVD is the liquid electrolytic conductivity as it limits its use to distilled water or non-conductive solutions. If the liquid contains too much charge carriers, the electric field cannot build up, meaning the plasma formation and stress confinement, driving the bubble formation, will not take place. This is shown in Fig. 9c, as the bubble radius starts decreasing at roughly $3 \mu \mathrm{S} / \mathrm{cm}$, as the ions are added to distilled water and stop producing bubbles altogether above $22 \mu \mathrm{S} / \mathrm{cm}$ for our setup at $100 \mu \mathrm{m}$ electrode gap. The discharge capability was tested with a $0.8 \mathrm{M}$ saccharose solutions, which might be useful for cellular interaction studies, offering a viable substitute for saline solutions, usually used for preventing osmotic shock on biological tissues. For LVD, however, we see that it produces bubbles throughout the range, and it is only at high conductivity values that gas bubbles were seen appearing along the electrodes, most likely due to electrolysis of water. This means a portion of the energy from the capacitor went to bubble formation, and a consequent drop of bubble radius is observed. Laser and TAM methods were not tested for liquid conductivity effects as the mechanism for bubble generation is not expected to be affected by this parameter.

Liquid temperature effects on bubble maximum radius, presented in Fig. 9d, show LVD bubbles getting larger with increasing temperature, while HVD bubbles do not vary significantly in the same temperature range. This is unexpected; as the vapor pressure of water increases with temperature, one would expect the bubble size to increase accordingly. Yet, an increase in the el. conductivity accompanying the temperature increase could be suppressing the bubble growth at the same time the vapor pressure increase is augmenting it, summing to roughly zero net effect. Interestingly, when the liquid temperature effects are observed, the variations in the normalized growth time for both LVD and HVD follow the same trend, even when the bubble radii do not. We cannot offer a satisfactory explanation for this observation. Moreover, in the HVD case the normalized growth times seem to be inversely linked to the bubble diameter, while for LVD the vapor pressure increase effects dominate. Figure 9d also shows discrepancies for the LVD bubble, as longer normalized growth times. Here the spark is typically observed throughout the growth period and is much longer than ns discharge typical for HVD. This is most likely the reason we observe the prolonged bubble growth period with this method. The discharge continues to heat the bubble content during growth, causing the expansion not to follow the timelines defined by the Rayleigh model for the growth and collapse of an empty void in infinite liquid. With proper equipment, the discharge times could be controlled and shortened to mimic energy impulses reminiscent to pulsed excitation techniques.

\subsection{Laser-induced method}

The benchmark technique, the laser-induced bubbles was not given as much scrutiny, as there are many reports on the topic in the literature. For the laser-generated bubbles, bubble volume is proportional to laser energy. Therefore, radius is expected to grow proportional to as a cube root of energy, while actual growth observed is slightly faster, implying that a slightly greater proportion of laser energy is absorbed in liquid at higher pulse energy. Bubbles generated by the method show high reproducibility in bubble size and lifetime, even if ordinary distilled water is used. Ultrapure water offers even slightly better conditions for bubble generation as impurities that could facilitate nucleation are removed, in turn avoiding small bubbles expanded by the negative pressure shockwave (Fig. 7g). Temperature effects on laser-induced bubbles was addressed in several previous studies and found the expected increase in the maximum radius with increased liquid temperature (Barbaglia and Bonetto 2004; Liu et al. 2011, 2013); however, one study found the same trend for near wall collapses and a decreasing $\mathrm{R}_{\max }$ with increasing temperature for bulk liquid bubbles (Zhang et al. 2019), while using a different laser wavelength. Overall, the laser-induced bubble generation is commonly used in the field as it provides reliable, versatile and close to perfect transient vapor bubbles. The bubble size range spans from about $10 \mu \mathrm{m}$ to a few $\mathrm{mm}$ and is easily tuneable by the applied pulse energy and optical densities filters if needed. 
The down side is the hazardous and expensive equipment required for bubble generation, as well as the limitation imposed by the optics working distance.

\section{Overview and recommendations}

The ranges of bubble radii and lifetimes that can be expected for the different methods are indicated by the color-coordinated arrows in Fig. 12. For individual techniques, the data are tabulated in Tables 1, 2, 3, 4. Care must be taken when comparing our result to those from the literature, as there are diverse bubble generation methods included. Also, due to ambiguous reporting of $\mathrm{R}_{\max }$, lifetimes and the experimental conditions, there are a few points placed below the predicted lifetime for the bubble size (dashed line), which is indicated for ambient pressure and $25{ }^{\circ} \mathrm{C}$. Either increased hydrostatic or system pressure will shorten bubble lifetimes with respect to its $R_{\max }$. For HVD, the bubble range spans almost three orders of magnitude. Low-power piezoelectric discharges, like the one used in our experimental setup, and the only similar method to our knowledge (Avila et al. 2015; Gonzalez-Avila et al. 2020), are showing comparable results. For high-power discharges, the techniques show accordance with the predicted lifetimes, even when large bubbles are produced. This does not hold for TAM, where the density fluctuations in the tube and the relatively long discharge, respectively, cause longer bubble lifetimes. From the results of Fig. 12, we can conclude that most of TAM users do not achieve Rayleigh-like collapse, while often reporting RPElike bubble radius evolution. The large laser-induced bubble from the literature falls far from the Rayleigh lifetime, as the reported dimensions and times are observed below ambient pressure. It is shown on the graph to demonstrate the higher range of the laser-induced bubbles that can be produced. For LVD, the collapse phase is in accordance with RPE, while shortening the discharge time could alleviate the growth phase elongation. For HVD and laser-induced bubble, however, the lifetimes are in agreement with the RPE predicted times as shown in Fig. 9b and more so in Figs. 11 and 12.

In Table 5, we can see and overview of the methods used to produce individual vapor bubbles. We focused on techniques able to produce spherical individual transient bubbles in bulk liquid; however, all of these methods could be used for near wall implosion studies. The pulsed laser-induced breakdown is the least intrusive and invasive method of the four, as the focusing elements may be outside the liquid reservoir, not disturbing the bubble dynamics. Furthermore, due to the absence of nuclei, it is likely that the induced bubbles stem from homogeneous nucleation, when ultrapure liquids are used. Their sphericity throughout the bubble lifetime is nearly perfect and bubble lifetimes are in accordance with theory for bubbles their size. However, it does require expensive equipment to obtain these "perfect" bubbles. Also, when considering the health risks associated with using high-power lasers, and the limitation placed on the technique due to the short working distances of the optics, it becomes clear that the laser-induced bubbles might not always be the optimal choice, when making single transient bubble experiments. The other three methods used all have either electrodes or the nucleation bubble rod, assuring heterogeneous nucleation. Considering electrode size compared to the bubble maximum size $(\sim \mathrm{mm}-\mathrm{cm})$ with the spark discharge techniques, it seems that the electrodes generally do not influence the sphericity of the bubble during the first growth and collapse. This is true until the very last phase of the collapse, where the solid surfaces finally cause the nonspherical collapse and jetting away from the electrodes, but therefore effecting the rebound bubble. The largest bubbles are produced with TAM (alongside high-powered HVD) and are more sensitive to perturbations, due to lower surface tension and the liquid motion relative to the stationary nucleation rod. This usually deforms the growing bubble and is perhaps the biggest shortfall of the technique. The simplest way to fix this problem is to mount the rod to the tube itself, as was done in our experimental setup, by doing so we lose an observation axis from below the tube. It could foreseeably be replaced altogether with a less intrusive way of generating nucleation sites (focused CW laser). An interesting solution was utilized by (Williams et al. 1997b) with the TAM; by introducing a small air bubble at the bottom and allowing it to rise to the target site, the method could function without the nucleation bubble rod. An important advantage of the TAM is its suitability to study temperature effects of the phase transition phenomenon. As cavitation is theoretically an isothermal process, there might be issues when energy deposition techniques are used, which can significantly heat the microenvironment around the formed bubble. Laser- and spark-induced bubbles at maximum radius are generally considered in thermodynamic equilibrium, and the bubble collapse dynamics are said not be affected by the initial energy deposition (Sato et al. 2013) and are therefore considered to be apt in most cases for cavitation research. However, the TAM should be considered when studying bubble generation and effects in the growth phase (Dular and Coutier-Delgosha 2013), as it does not rely on a concentrated energy deposition initiating the growth. Furthermore, energy deposition techniques, which are based on initial plasma expansion, should generally not be used for studying chemical production of cavitation bubbles due to inherent radical and active chemical species production of the plasma (Sato et al. 2013). Although most radicals by their nature do not have long halflives, they can survive for times in the range of the bubble lifetime. Moreover, radical recombination can form stable reactive species, like hydrogen peroxide, which can outlive bubble lifetimes by many timescales. 


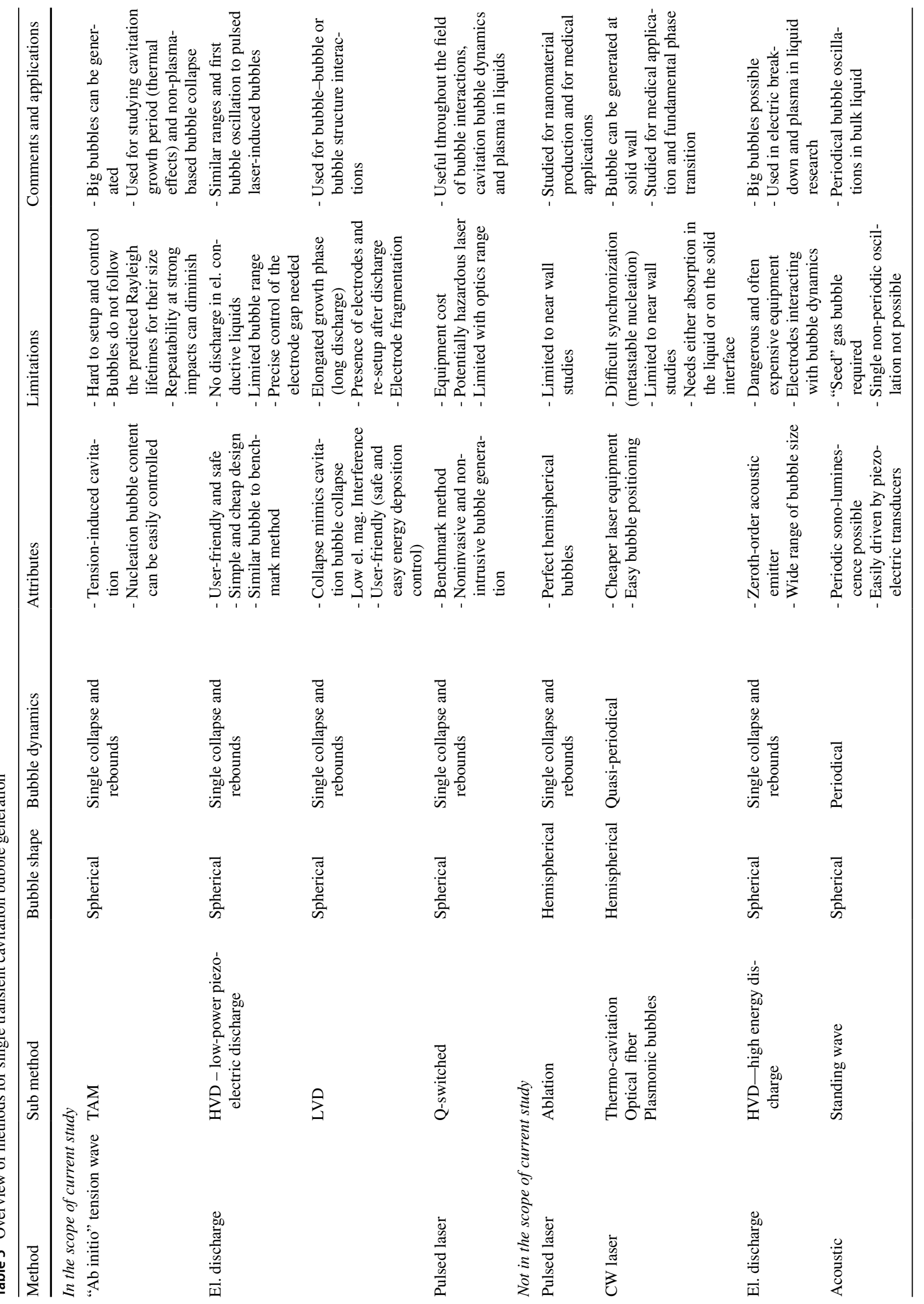


When bubble size control and/or simultaneous multiple bubble generation is key to the experimental setup, then perhaps the use of the LVD technique might outweigh its shortfalls. Without controllable discharge times, the bubble growth in LVD techniques deviates from RPE bubble dynamics. Electrode breakup in each discharge can become tedious for the repositioning and can affect reproducibility. Less electromagnetic interference (Goh et al. 2013; Zhang et al. 2018), ease of bubble size control, user safety and multi-electrode discharge possibilities are the positive attributes of these technique. Also, if the method is not properly designed it can suffer from mechanical switching problems, which can lead to problems with reproducibility due to the contact bounce effect. With the use of a MOSFET, we can eliminate drawbacks of mechanical switching, while also taking advantage of the fast opening (Goh et al. 2013) and potentially pulse time regulation. As the piezoelectric HVD used in this article and laser-induced techniques produce bubbles following the RPE and also roughly the same size, they could be interchangeable in some cases if the water conductivity is in the discharging range of HVD. Both bubbles originate from plasma-generated nucleus and continue with almost identical dynamics to the end of the first oscillation, after which the similarities end. A drastically cheaper and safer setup, with easy implementation, as well as comparable reproducibility makes the use of piezoelectric sparkers a viable option; however, for precise bubble size control a micropositioning system is needed.

Several other options are available when hemispherical bubbles and periodic bubble excitation is viable. These are also presented in Table 5. When pulsed lasers are focused on a solid surface, the plasma will be formed on the interface, often accompanied by ablation of the solid material. This produces a hemispherical bubble, following the RPE, and is often studied for nanomaterial production (Lam et al. 2016). CW lasers can be used with many methods to heat up the liquid to the spinodal limit and induce nucleation. This is used for medical applications and for basic research of bubble nucleation and growth; however, in addition to forming hemispherical bubbles it is also a quasi-periodic, as the bubbles will repeatedly nucleate as long as the $\mathrm{CW}$ laser is applied to the target. Also, as the spinodal temperatures are reached quickly with this method, the metastability of water introduces an element of randomness to the nucleation, so precise control and synchronization can be problematic. Spherical bubbles can be trapped by antinodes in acoustic fields, but by its nature the excitation will be periodic, not transient. Although some interesting insights have been discovered by studying plasma in the collapse phase of stable gas bubble oscillations showing periodic sonoluminescent properties, this technique is generally not used for studying bubble interactions, as positioning of the bubbles is difficult if not impossible. The high-power HVD discharges are also a good source for studying plasma in liquids and electrical breakdown of liquids. Spherical bubbles can be produced and have been shown to be good zeroth-order acoustic emitters, but due to the large bubbles produced, it can be difficult to control the shape as the surface tension effects lessen, which can affect sphericity. Also, the high-power voltage equipment expensive and heightened protective measures have to be used. Overall, the methods in Table 5, not covered in the scope of the study, either are not able to produce spherical transient bubbles or are impractical for such applications. As we show in our work, each of the techniques has its positive and negative aspects. It remains at the discretion of the user to choose the correct one, based on the limitation of the experimental setup and the physics of the phenomenon being explored.

\section{Conclusions}

Four vapor bubble generation methods were tested for their governing parameters, as well as the bubble response to liquid temperature and electrolytic conductivity. General overview of the mechanisms behind each bubble generation technique along with examples of simple and effective methods for vapor bubble generation are presented in detail. The bubble production repeatability and the sphericity throughout the bubble lifetime are evaluated for each method. Bubble lifetime in accordance with the Rayleigh model and the isochronal conditions of the growth and collapse period are evaluated. Results for each method were compared to the results from similar experimental techniques in the literature, giving the characteristic range of bubble sizes and lifetimes expected for each technique. Laser and low-energy high-voltage discharge bubbles found to be Rayleigh-like throughout their lifetime, while low-voltage discharge and the tube arrest method can both suffer from elongated growth periods. Energy deposition-based techniques are also simple to implement for studies involving bubble dynamics and bubble-structure interactions. However, bubbles generated by energy deposition, based on initial plasma generation are not good approximation for cavitation bubble chemical production, due to the initial plasma. Thermal effect studies should not be based on these methods as well, but rather on tension-based techniques, like the tube arrest method, to avoid the temperature perturbation from the initial energy pulse. We also suggest future improvements for the techniques used, to allow the reader to make an informed decision on the experimental setup, when studying single cavitation bubble dynamics.

Acknowledgements The authors acknowledge the financial support from the Slovenian Research Agency (research core Funding No. P2-0270, P2-0401 and project No. L2-9240) and the European 
Research Council (ERC) under the European Union's Framework Program for research and innovation, Horizon 2020 (Grant agreement ${ }^{\circ}$ 771567 - CABUM). The authors would also like to thank Tone Godeša from the Faculty of mechanical engineering, University of Ljubljana, for help with the experimental setup.

Open Access This article is licensed under a Creative Commons Attribution 4.0 International License, which permits use, sharing, adaptation, distribution and reproduction in any medium or format, as long as you give appropriate credit to the original author(s) and the source, provide a link to the Creative Commons licence, and indicate if changes were made. The images or other third party material in this article are included in the article's Creative Commons licence, unless indicated otherwise in a credit line to the material. If material is not included in the article's Creative Commons licence and your intended use is not permitted by statutory regulation or exceeds the permitted use, you will need to obtain permission directly from the copyright holder. To view a copy of this licence, visit http://creativecommons.org/licenses/by/4.0/.

\section{References}

Akhatov I, Lindau O, Topolnikov A et al (2001) Collapse and rebound of a laser-induced cavitation bubble. Phys Fluids 13:2805-2819. https://doi.org/10.1063/1.1401810

Andersen A, Mørch KA (2015) Cavitation nuclei in water exposed to transient pressures. J Fluid Mech 771:424-448. https://doi.org/ 10.1017/jfm.2015.185

Ando K, Liu A-Q, Ohl C-D (2012) Homogeneous nucleation in water in microfluidic channels. Phys Rev Lett 109:044501. https://doi. org/10.1103/PhysRevLett.109.044501

Arora M, Ohl CD, Lohse D (2007) Effect of nuclei concentration on cavitation cluster dynamics. J Acoust Soc Am 121:3432-3436. https://doi.org/10.1121/1.2722045

Askar'yan GA, Prokhorov AM, Chantuviya GF, Shipulo GP (1963) The effects of a laser beam in a liquid. J Exp Theor Phys 17:1463

Atrazhev VM, Vorob'ev VS, Timoshkin IV, et al (2010) Mechanisms of impulse breakdown in liquid: the role of joule heating and formation of gas cavities. IEEE Trans Plasma Sci 38:2644-2651. https://doi.org/10.1109/TPS.2010.2046337

Avila SRG, Song C, Ohl C-D (2015) Fast transient microjets induced by hemispherical cavitation bubbles. J Fluid Mech 767:31-51. https://doi.org/10.1017/jfm.2015.33

Azouzi MEM, Ramboz C, Lenain J-F, Caupin F (2013) A coherent picture of water at extreme negative pressure. Nat Phys 9:38-41. https://doi.org/10.1038/nphys 2475

Baird MHI (1963) Resonant bubbles in a vertically vibrating liquid column. Can J Chem Eng 41:52-55. https://doi.org/10.1002/cjce. 5450410204

Barbaglia MO, Bonetto FJ (2004) Dependence on liquid temperature and purity of light emission characteristics in single cavitation bubble luminescence. J Appl Phys 95:1756-1759. https://doi. org/10.1063/1.1637711

Bergant A, Simpson AR, Tijsseling AS (2006) Water hammer with column separation : a historical review. J Fluids Struct 22:135-171. https://doi.org/10.1016/j.jfluidstructs.2005.08.008

Brennen CE (1995) Cavitation and Bubble Dynamics. Oxford University Press, New York

Briggs LJ (1950) Limiting negative pressure of water. J Appl Phys 21:721-722. https://doi.org/10.1063/1.1699741

Bruggeman PJ, Kushner MJ, Locke BR et al (2016) Plasma-liquid interactions: a review and roadmap. Plasma Sources Sci Technol 25:053002. https://doi.org/10.1088/0963-0252/25/5/053002
Brujan E-A (2008) Shock wave emission from laser-induced cavitation bubbles in polymer solutions. Ultrasonics 48:423-426. https:// doi.org/10.1016/j.ultras.2008.02.001

Brujan E-A, Vogel A (2006) Stress wave emission and cavitation bubble dynamics by nanosecond optical breakdown in a tissue phantom. J Fluid Mech 558:281-308. https://doi.org/10.1017/S0022 112006000115

Buchanan RH, Jameson G, Oedjoe D (1962) Cyclic migration of bubbles in vertically vibrating liquid columns. Ind Eng Chem Fundam 1:82-86. https://doi.org/10.1021/i160002a003

Buogo S, Cannelli GB (2002) Implosion of an underwater spark-generated bubble and acoustic energy evaluation using the Rayleigh model. J Acoust Soc Am 111:2594-2600. https://doi.org/10. $1121 / 1.1476919$

Buogo S, Plocek J, Vokurka K (2009) Efficiency of Energy Conversion in Underwater Spark Discharges and Associated Bubble Oscillations: Experimental Results. https://www.ingentacon nect.com/content/dav/aaua/2009/00000095/00000001/art00 004. Accessed 23 May 2019

Cannelli GB, D’Ottavi E, Prosperetti A (1990) Bubble Activity Induced By High-power Marine Sources. In: Conference Proceedings on Engineering in the Ocean Environment. pp 533-537

Caupin F, Herbert E (2006) Cavitation in water: a review. Comptes Rendus Phys 7:1000-1017. https://doi.org/10.1016/j.crhy. 2006.10.015

Chen Q-D, Wang L (2005) Luminescence from transient cavitation bubbles in water. Phys Lett A 339:110-117. https://doi.org/10. 1016/j.physleta.2005.03.029

Chen Q, Li J, Li Y (2015) A review of plasma-liquid interactions for nanomaterial synthesis. J Phys Appl Phys 48:424005. https:// doi.org/10.1088/0022-3727/48/42/424005

Chesterman WD (1952) The dynamics of small transient cavities. Proc Phys Soc Sect B 65:846-858. https://doi.org/10.1088/ 0370-1301/65/11/302

Chong-Fu Y, Chao L, De-Long X, Jing-Jun D (2008) The pressure field in the liquid column in the tube-arrest method. Chin Phys B 17:2580-2589. https://doi.org/10.1088/1674-1056/17/7/040

Crum LA (2015) Resource paper: sonoluminescence. J Acoust Soc Am 138:2181-2205. https://doi.org/10.1121/1.4929687

Daily J, Pendlebury J, Langley K et al (2014) Catastrophic cracking courtesy of quiescent cavitation. Phys Fluids 26:091107. https://doi.org/10.1063/1.4894073

Dijkink R, Ohl C-D (2008) Laser-induced cavitation based micropump. Lab Chip 8:1676-1681. https://doi.org/10.1039/b8069 $12 \mathrm{c}$

Dular M, Coutier-Delgosha O (2013) Thermodynamic effects during growth and collapse of a single cavitation bubble. J Fluid Mech 736:44-66. https://doi.org/10.1017/jfm.2013.525

Dular M, Petkovšek M (2015) New insights into the mechanisms of cavitation erosion. J Phys Conf Ser 656:012046. https://doi.org/ 10.1088/1742-6596/656/1/012046

Dular M, Griessler-Bulc T, Gutierrez-Aguirre I et al (2016) Use of hydrodynamic cavitation in (waste)water treatment. Ultrason Sonochem 29:577-588. https://doi.org/10.1016/j.ultsonch.2015. 10.010

Dular M, Požar T, Zevnik J, Petkovšek R (2019) High speed observation of damage created by a collapse of a single cavitation bubble. Wear 418-419:13-23. https://doi.org/10.1016/j.wear. 2018.11.004

F.R.S LROM, (1917) VIII. On the pressure developed in a liquid during the collapse of a spherical cavity. Lond Edinb Dublin Philos Mag J Sci 34:94-98. https://doi.org/10.1080/14786440808635681

Fong SW, Adhikari D, Klaseboer E, Khoo BC (2009) Interactions of multiple spark-generated bubbles with phase differences. Exp Fluids 46:705-724. https://doi.org/10.1007/s00348-008-0603-4 
Fry LH, Adair P, Williams R (1999) Long life sparker for pulse powered underwater acoustic transducer. In: Digest of Technical Papers. 12th IEEE International Pulsed Power Conference. (Cat. No.99CH36358). pp 781-784 vol.2

Fujikawa S, Akamatsu T (1978) Experimental investigations of cavitation bubble collapse by a water shock tube. JSME Int J Ser B 21:223-230

Futakawa M, Naoe T, Kogawa H et al (2014) Cavitation erosion induced by proton beam bombarding mercury target for highpower spallation neutron sources. Exp Therm Fluid Sci 57:365370. https://doi.org/10.1016/j.expthermflusci.2014.05.014

Gaggol M, Przyjazny A, Boczkaj G (2018) Wastewater treatment by means of advanced oxidation processes based on cavitation-A review. Chem Eng J. https://doi.org/10.1016/j.cej.2018.01.049

Goh BHT, Oh YDA, Klaseboer E et al (2013) A low-voltage sparkdischarge method for generation of consistent oscillating bubbles. Rev Sci Instrum 84:014705. https://doi.org/10.1063/1. 4776187

Gong SW, Goh BHT, Ohl SW, Khoo BC (2012) Interaction of a sparkgenerated bubble with a rubber beam: numerical and experimental study. Phys Rev E 86:026307. https://doi.org/10.1103/PhysR evE.86.026307

Gonzalez-Avila SR, van Blokland AC, Zeng Q, Ohl C-D (2020) Jetting and shear stress enhancement from cavitation bubbles collapsing in a narrow gap. J Fluid Mech. https://doi.org/10.1017/jfm. 2019.938

Grieser F, Choi P-K, Enomoto N et al (2015) Sonochemistry and the acoustic bubble. Elsevier

Hamdan A, Černevičs K, Cha MS (2017) The effect of electrical conductivity on nanosecond discharges in distilled water and in methanol with argon bubbles. J Phys Appl Phys 50:185207. https://doi.org/10.1088/1361-6463/aa6969

Harrison M (1952) An experimental study of single bubble cavitation noise. J Acoust Soc Am 24:776-782. https://doi.org/10.1121/1. 1906978

Hellman AN, Rau KR, Yoon HH et al (2007) Laser-induced mixing in microfluidic channels. Anal Chem 79:4484-4492. https://doi. org/10.1021/ac070081i

Horikoshi S, Serpone N (2017) In-liquid plasma: a novel tool in the fabrication of nanomaterials and in the treatment of wastewaters. RSC Adv 7:47196-47218. https://doi.org/10.1039/C7RA09600C

Horvat D, Orthaber U, Schille J et al (2018) Laser-induced bubble dynamics inside and near a gap between a rigid boundary and an elastic membrane. Int J Multiph Flow 100:119-126. https:// doi.org/10.1016/j.ijmultiphaseflow.2017.12.010

Huang Y, Yan H, Wang B et al (2014) The electro-acoustic transition process of pulsed corona discharge in conductive water. J Phys Appl Phys 47:255204. https://doi.org/10.1088/0022-3727/47/25/ 255204

Huang Y, Zhang L, Zhang X et al (2015) Electroacoustic process study of plasma sparker under different water depth. IEEE J Ocean Eng 40:947-956. https://doi.org/10.1109/JOE.2014.2382451

Ikeda T, Yoshizawa S, Koizumi N et al (2016) Focused Ultrasound and Lithotripsy. In: Escoffre J-M, Bouakaz A (eds) Therapeutic Ultrasound. Springer International Publishing, Cham, pp 113-129

Iosilevskii G, Weihs D (2008) Speed limits on swimming of fishes and cetaceans. J R Soc Interface 5:329-338. https://doi.org/10.1098/ rsif.2007.1073

Jiang B, Zheng J, Qiu S et al (2014) Review on electrical discharge plasma technology for wastewater remediation. Chem Eng J 236:348-368. https://doi.org/10.1016/j.cej.2013.09.090

Jones WM, Overton GDN, Trevena DH (1981) Tensile strength experiments with water using a new type of Berthelot tube. J Phys Appl Phys 14:1283-1291. https://doi.org/10.1088/0022-3727/14/7/016
Kannan YS, Karri B, Sahu KC (2018) Letter: Entrapment and interaction of an air bubble with an oscillating cavitation bubble. Phys Fluids 30:041701. https://doi.org/10.1063/1.5025122

Kapahi A, Hsiao C-T, Chahine GL (2015) Shock-induced bubble collapse versus rayleigh collapse. J Phys Conf Ser 656:012128. https://doi.org/10.1088/1742-6596/656/1/012128

Khoo BC, Adikhari D, Fong SW, Klaseboer E (2009) Multiple sparkgenerated bubble interactions. Mod Phys Lett B 23:229-232. https://doi.org/10.1142/S0217984909018072

Kiyama A, Tagawa Y, Ando K, Kameda M (2015) Effects of a water hammer and cavitation on jet formation in a test tube. J Fluid Mech 787:224-236. https://doi.org/10.1017/jfm.2015.690

Kosel J, Gutiérrez-Aguirre I, Rački N et al (2017) Efficient inactivation of MS-2 virus in water by hydrodynamic cavitation. Water Res 124:465-471. https://doi.org/10.1016/j.watres.2017.07.077

Kudryashov NA, Sinelshchikov DI (2014) Analytical solutions of the Rayleigh equation for empty and gas-filled bubble. J Phys Math Theor 47:405202. https://doi.org/10.1088/1751-8113/47/40/ 405202

Kudryashov NA, Sinelshchikov DI (2015) Analytical solutions for problems of bubble dynamics. Phys Lett A 379:798-802. https:// doi.org/10.1016/j.physleta.2014.12.049

Lajoinie G, De Cock I, Coussios CC et al (2016) In vitro methods to study bubble-cell interactions: fundamentals and therapeutic applications. Biomicrofluidics. https://doi.org/10.1063/1.49404 29

Lam J, Lombard J, Dujardin C et al (2016) Dynamical study of bubble expansion following laser ablation in liquids. Appl Phys Lett 108:074104. https://doi.org/10.1063/1.4942389

Lazic V, Jovićević S (2014) Laser induced breakdown spectroscopy inside liquids: processes and analytical aspects. Spectrochim Acta Part B at Spectrosc 101:288-311. https://doi.org/10.1016/j. sab.2014.09.006

Leighton TG (2007) Derivation of the Rayleigh-Plesset Equation in Terms of Volume. University of Southampton

Lew KSF, Klaseboer E, Khoo BC (2007) A collapsing bubble-induced micropump: an experimental study. Sens Actuators Phys 133:161-172. https://doi.org/10.1016/j.sna.2006.03.023

Lewis TJ (1994) Basic electrical processes in dielectric liquids. IEEE Trans Dielectr Electr Insul 1:630-643. https://doi.org/10.1109/ 94.311706

Lewis TJ (1996) New electro-mechanical concepts of the primary mechanisms of electrical breakdown in liquids. In: ICDL'96. 12th International Conference on Conduction and Breakdown in Dielectric Liquids. pp 273-278

Li F, Yuan F, Sankin G et al (2017) A microfluidic system with surface patterning for investigating cavitation bubble(s)-cell interaction and the resultant bioeffects at the single-cell level. J vis Exp JoVE. https://doi.org/10.3791/55106

Liu X, Hou Y, Liu X et al (2011) Oscillation characteristics of a laserinduced cavitation bubble in water at different temperatures. Optik 122:1254-1257. https://doi.org/10.1016/j.ijleo.2010.08. 010

Liu X, Long Z, He J et al (2013) Experimental study of temperature effect on the growth and collapse of cavitation bubbles near a rigid boundary. Optoelectron Lett 9:317-320. https://doi.org/10. 1007/s11801-013-2422-y

Locke B (2012) Environmental applications of electrical discharge plasma with liquid water-A mini review. Int J Plasma Environ Sci Technol 6:194-203

Luo X, Ji B, Tsujimoto Y (2016) A review of cavitation in hydraulic machinery. J Hydrodyn Ser B 28:335-358. https://doi.org/10. 1016/S1001-6058(16)60638-8

Luo J, Xu W, Deng J et al (2018) Experimental study on the impact characteristics of cavitation bubble collapse on a wall. Water 10:1262. https://doi.org/10.3390/w10091262 
Mancas SC, Rosu HC (2016) Evolution of spherical cavitation bubbles: parametric and closed-form solutions. Phys Fluids 28:022009. https://doi.org/10.1063/1.4942237

Mellen RH (1956) An experimental study of the collapse of a spherical cavity in water. J Acoust Soc Am 28:447-454. https://doi.org/10. $1121 / 1.1908354$

Mohammadzadeh M, Gonzalez-Avila SR, Wan YC et al (2016) Photoacoustic shock wave emission and cavitation from structured optical fiber tips. Appl Phys Lett 108:024101. https://doi.org/ $10.1063 / 1.4939511$

Nikitenko SI, Pflieger R (2017) Toward a new paradigm for sonochemistry: short review on nonequilibrium plasma observations by means of MBSL spectroscopy in aqueous solutions. Ultrason Sonochem 35:623-630. https://doi.org/10.1016/j.ultsonch.2016. 02.003

Obreschkow D, Bruderer M, Farhat M (2012) Analytical approximations for the collapse of an empty spherical bubble. Phys Rev E Stat Nonlin Soft Matter Phys 85:066303. https://doi.org/10. 1103/PhysRevE.85.066303

Oguri R, Ando K (2018) Cavitation bubble nucleation induced by shock-bubble interaction in a gelatin gel. Phys Fluids 30:051904. https://doi.org/10.1063/1.5026713

Ohl CD (2002a) Cavitation inception following shock wave passage. Phys Fluids 14:3512-3521. https://doi.org/10.1063/1.1503351

Ohl C-D (2002b) Probing luminescence from nonspherical bubble collapse. Phys Fluids 14:2700-2708. https://doi.org/10.1063/1. 1489682

Ohl CD, Ikink R (2003) Shock-wave-induced jetting of micron-size bubbles. Phys Rev Lett 90:214502. https://doi.org/10.1103/ PhysRevLett.90.214502

Orthaber U, Zevnik J, Petkovšek R, Dular M (2020) Cavitation bubble collapse in a vicinity of a liquid-liquid interface-basic research into emulsification process. Ultrason Sonochem 68:105224. https://doi.org/10.1016/j.ultsonch.2020.105224

Overton GDN, Edwards MJ, Trevena DH (1982) Dissolved gas content and the static breaking tension of water. J Phys Appl Phys 15:L129-L131. https://doi.org/10.1088/0022-3727/15/10/003

Overton GDN, Williams PR, Trevena DH (1984) The influence of cavitation history and entrained gas on liquid tensile strength. J Phys Appl Phys 17:979-987. https://doi.org/10.1088/00223727/17/5/012

Padilla-Martinez JP, Berrospe-Rodriguez C, Aguilar G et al (2014) Optic cavitation with $\mathrm{CW}$ lasers: a review. Phys Fluids 26:122007. https://doi.org/10.1063/1.4904718

Pain A, Hui Terence Goh B, Klaseboer E et al (2012) Jets in quiescent bubbles caused by a nearby oscillating bubble. J Appl Phys 111:054912. https://doi.org/10.1063/1.3692749

Pan Z, Kiyama A, Tagawa Y et al (2017) Cavitation onset caused by acceleration. Proc Natl Acad Sci U S A 114:8470-8474. https://doi.org/10.1073/pnas.1702502114

Patek SN, Caldwell RL (2005) Extreme impact and cavitation forces of a biological hammer: strike forces of the peacock mantis shrimp Odontodactylus scyllarus. J Exp Biol 208:3655-3664. https://doi.org/10.1242/jeb.01831

Podbevsek D, Colombet D, Ledoux G, Ayela F (2018) Observation of chemiluminescence induced by hydrodynamic cavitation in microchannels. Ultrason Sonochem 43:175-183. https://doi. org/10.1016/j.ultsonch.2018.01.004

Podbevšek D, Colombet D, Ayela F, Ledoux G (2021) Localization and quantification of radical production in cavitating flows with luminol chemiluminescent reactions. Ultrason Sonochem 71:105370. https://doi.org/10.1016/j.ultsonch.2020.105370

Pongrác B, Šimek M, Ondáč P et al (2019) Velocity of initial propagation of positive nanosecond discharge in liquid water: dependence on high voltage amplitude and water conductivity.
Plasma Sources Sci Technol. https://doi.org/10.1088/13616595/aae91f

Prosperetti A (1982) A generalization of the Rayleigh-Plesset equation of bubble dynamics. Phys Fluids 25:409-410. https://doi. org/10.1063/1.863775

Qi-Dai C, Long W (2004) Production of large size single transient cavitation bubbles with tube arrest method. Chin Phys 13:564570. https://doi.org/10.1088/1009-1963/13/4/028

Qi-Dai CHEN, WL, CHEN Qi-Dai WL, (2004) Luminescence from Tube-Arrest Bubbles in Pure Glycerin. Chin Phys Lett 21:1822-1824

Qiu X, Bouchiat V, Colombet D, Ayela F (2019) Liquid-phase exfoliation of graphite into graphene nanosheets in a hydrocavitating 'lab-on-a-chip.' RSC Adv 9:3232-3238. https://doi.org/ 10.1039/C8RA05976D

Quinto-Su PA, Venugopalan V, Ohl CD (2008) Generation of laserinduced cavitation bubbles with a digital hologram. Opt Express 16:18964-18969. https://doi.org/10.1364/OE.16.018964

Quinto-Su PA, Lim KY, Ohl C-D (2009) Cavitation bubble dynamics in microfluidic gaps of variable height. Phys Rev E Stat Nonlin Soft Matter Phys 80:047301. https://doi.org/10.1103/PhysRevE. 80.047301

Quinto-Su PA, Suzuki M, Ohl C-D (2014) Fast temperature measurement following single laser-induced cavitation inside a microfluidic gap. Sci Rep 4:5445. https://doi.org/10.1038/srep05445

Reich S, Schönfeld P, Wagener P et al (2017) Pulsed laser ablation in liquids: impact of the bubble dynamics on particle formation. J Colloid Interface Sci 489:106-113. https://doi.org/10.1016/j. jcis.2016.08.030

Richards BE, Trevena DH, Edwards DH (1980) Cavitation experiments using a water shock tube. J Phys Appl Phys 13:1315-1323. https://doi.org/10.1088/0022-3727/13/7/027

Rodríguez-Rodríguez J, Casado-Chacón A, Fuster D (2014) Physics of beer tapping. Phys Rev Lett 113:214501. https://doi.org/10.1103/ PhysRevLett.113.214501

Rond C, Desse JM, Fagnon N et al (2018) Time-resolved diagnostics of a pin-to-pin pulsed discharge in water: pre-breakdown and breakdown analysis. J Phys Appl Phys 51:335201. https://doi. org/10.1088/1361-6463/aad175

Rosselló JM, Urteaga R, Bonetto FJ (2018) A novel water hammer device designed to produce controlled bubble collapses. Exp Therm Fluid Sci 92:46-55. https://doi.org/10.1016/j.expthermfl usci.2017.11.016

Sajjadi B, Abdul Aziz AR, Ibrahim S (2015) Mechanistic analysis of cavitation assisted transesterification on biodiesel characteristics. Ultrason Sonochem 22:463-473. https://doi.org/10.1016/j.ultso nch.2014.06.004

Šarc A, Oder M, Dular M (2016) Can rapid pressure decrease induced by supercavitation efficiently eradicate Legionella pneumophila bacteria? Desalination Water Treat 57:2184-2194. https://doi. org/10.1080/19443994.2014.979240

Sato T, Tinguely M, Oizumi M, Farhat M (2013) Evidence for hydrogen generation in laser- or spark-induced cavitation bubbles. Appl Phys Lett 102:074105. https://doi.org/10.1063/1.4793193

Schmid J (1959) Kinematographische Untersuchung der EinzelblasenKavitation. https://www.ingentaconnect.com/content/dav/aaua/ 1959/00000009/00000004/art00006? crawler=true. Accessed 21 Jul 2019

Shima A, Takayama K, Tomita Y, Ohsawa N (1983) Mechanism of impact pressure generation from spark-generated bubble collapse near a wall. AIAA J 21:55-59. https://doi.org/10.2514/3.8027

Sinibaldi G, Occhicone A, Alves Pereira F et al (2019) Laser induced cavitation: plasma generation and breakdown shockwave. Phys Fluids 31:103302. https://doi.org/10.1063/1.5119794 
Song WD, Hong MH, Lukyanchuk B, Chong TC (2004) Laser-induced cavitation bubbles for cleaning of solid surfaces. J Appl Phys 95:2952-2956. https://doi.org/10.1063/1.1650531

Stride EP, Coussios CC (2010) Cavitation and contrast: the use of bubbles in ultrasound imaging and therapy. Proc Inst Mech Eng [h] 224:171-191. https://doi.org/10.1243/09544119JEIM622

Su C-K, Camara C, Kappus B, Putterman SJ (2003) Cavitation luminescence in a water hammer: upscaling sonoluminescence. Phys Fluids 15:1457-1461. https://doi.org/10.1063/1.1572493

Sun Y, Gao Y, Yan P, et al (2009) Development of a $20 \mathrm{~kJ}$ Sparker for High Resolution Ocean Seismic Survey. Acta Phys Pol A. https:// doi.org/10.12693/APhysPolA.115.1059

Sun Y, Timoshkin IV, Given MJ et al (2016) Impulsive discharges in water: acoustic and hydrodynamic parameters. IEEE Trans Plasma Sci 44:2156-2166. https://doi.org/10.1109/TPS.2016. 2583066

Sunka P, Babicky V, Clupek M et al (2004) Localized damage of tissues induced by focused shock waves. IEEE Trans Plasma Sci 32:16091613. https://doi.org/10.1109/TPS.2004.830965

Supponen O, Obreschkow D, Kobel P et al (2019) Detailed experiments on weakly deformed cavitation bubbles. Exp Fluids 60:33. https:// doi.org/10.1007/s00348-019-2679-4

Suslick KS, Flannigan DJ (2008) Inside a collapsing bubble: sonoluminescence and the conditions during cavitation. Annu Rev Phys Chem 59:659-683. https://doi.org/10.1146/annurev.physchem.59. 032607.093739

Taleyarkhan RP, West CD, Cho JS et al (2002) Evidence for nuclear emissions during acoustic cavitation. Science 295:1868-1873. https:// doi.org/10.1126/science.1067589

Timoshkin IV, Fouracre RA, Given MJ, MacGregor SJ (2006) Hydrodynamic modelling of transient cavities in fluids generated by high voltage spark discharges. J Phys Appl Phys 39:4808-4817. https:// doi.org/10.1088/0022-3727/39/22/011

Tinguely M, Obreschkow D, Kobel P et al (2012) Energy partition at the collapse of spherical cavitation bubbles. Phys Rev E 86:046315. https://doi.org/10.1103/PhysRevE.86.046315

Trevena DH (1984) Cavitation and the generation of tension in liquids. J Phys Appl Phys 17:2139-2164. https://doi.org/10.1088/0022-3727/ 17/11/003

Turangan CK, Ong GP, Klaseboer E, Khoo BC (2006) Experimental and numerical study of transient bubble-elastic membrane interaction. J Appl Phys 100:054910. https://doi.org/10.1063/1.2338125

Verhaagen B, Fernández Rivas D (2016) Measuring cavitation and its cleaning effect. Ultrason Sonochem 29:619-628. https://doi.org/ 10.1016/j.ultsonch.2015.03.009

Vilagrosa A, Chirino E, Peguero-Pina JJ et al (2012) Xylem Cavitation and Embolism in Plants Living in Water-Limited Ecosystems. Plant Responses to Drought Stress. Springer, Berlin, Heidelberg, pp 63-109

Vogel A, Venugopalan V (2003) Mechanisms of pulsed laser ablation of biological tissues. Chem Rev 103:577-644. https://doi.org/10. $1021 / \mathrm{cr} 010379 n$

Vogel A, Busch S, Jungnickel K, Birngruber R (1994) Mechanisms of intraocular photodisruption with picosecond and nanosecond laser pulses. Lasers Surg Med 15:32-43. https://doi.org/10.1002/lsm. 1900150106

Vogel A, Busch S, Parlitz U (1996) Shock wave emission and cavitation bubble generation by picosecond and nanosecond optical breakdown in water. J Acoust Soc Am 100:148-165. https://doi.org/10. $1121 / 1.415878$

Vogel A, Noack J, Nahen K et al (1999) Energy balance of optical breakdown in water at nanosecond to femtosecond time scales. Appl Phys B 68:271-280. https://doi.org/10.1007/s003400050617

Vokurka K (1988) Evaluation of data from experiments with spark and laser generated bubbles. Czechoslov J Phys 38:35-46. https://doi. org/10.1007/BF01596517
Wang Y, Zaytsev ME, The HL et al (2017) Vapor and Gas-bubble growth dynamics around laser-irradiated, water-immersed plasmonic nanoparticles. ACS Nano 11:2045-2051. https://doi.org/10.1021/acsna no.6b08229

Wang Y, Zaytsev ME, Lajoinie G et al (2018) Giant and explosive plasmonic bubbles by delayed nucleation. Proc Natl Acad Sci 115:7676-7681. https://doi.org/10.1073/pnas.1805912115

Williams PR, Williams RL (2004) Cavitation and the tensile strength of liquids under dynamic stressing. Mol Phys 102:2091-2102. https:// doi.org/10.1080/00268970412331292786

Williams PR, Williams RL (2002) Cavitation of liquids under dynamic stressing by pulses of tension. J Phys Appl Phys 35:2222-2230. https://doi.org/10.1088/0022-3727/35/17/321

Williams PR, Williams PM, Brown SWJ (1997a) Pressure waves arising from the oscillation of cavitation bubbles under dynamic stressing. J Phys Appl Phys 30:1197-1206. https://doi.org/10.1088/ 0022-3727/30/8/007

Williams PR, Williams PM, Brown SWJ (1997b) A technique for studying liquid jets formed by cavitation bubble collapse under shockwaves, near a free surface. J Non-Newton Fluid Mech 72:101-110. https://doi.org/10.1016/S0377-0257(97)00020-7

Williams PR, Williams PM, Brown SWJ (1998) An instrument for studying cavitation phenomena in liquids subjected to tension generatedab initioand by free-surface reflection of compressional waves. Meas Sci Technol 9:976-982. https://doi.org/10.1088/0957-0233/9/ 6/015

Williams PR, Williams PM, Brown SWJ, Temperley HNV (1999) On the tensile strength of water under pulsed dynamic stressing. Proc $\mathrm{R}$ Soc Lond Ser Math Phys Eng Sci 455:3311-3323. https://doi.org/ 10.1098/rspa.1999.0452

Wu X-M, Ying C-F, Li C (2005) Luminescence of transient single cavitation bubbles in non-aqueous liquids produced by the modified tube-arrest method. Chin Phys 14:999-1005. https://doi.org/10. 1088/1009-1963/14/5/025

$\mathrm{Xu} \mathrm{W,} \mathrm{Zhai} \mathrm{Y,} \mathrm{Luo} \mathrm{J} \mathrm{et} \mathrm{al} \mathrm{(2019)} \mathrm{Experimental} \mathrm{study} \mathrm{of} \mathrm{the} \mathrm{influence} \mathrm{of}$ flexible boundaries with different elastic moduli on cavitation bubbles. Exp Therm Fluid Sci 109:109897. https://doi.org/10.1016/j. expthermflusci.2019.109897

Yan Z, Chrisey DB (2012) Pulsed laser ablation in liquid for micro-/nanostructure generation. J Photochem Photobiol C Photochem Rev 13:204-223. https://doi.org/10.1016/j.jphotochemrev.2012.04.004

Yasui K (2018) Acoustic Cavitation. Acoustic Cavitation and Bubble Dynamics. Springer, Cham, pp 1-35

Young FR (1999) Cavitation. Imperial College Press

Zaytsev ME, Wang Y, Zhang Y et al (2020) Gas-vapor interplay in plasmonic bubble shrinkage. J Phys Chem C 124:5861-5869. https:// doi.org/10.1021/acs.jpcc.9b10675

Zhang LC, Zhu XL, Huang YF et al (2016a) Development of a simple model for predicting the spark-induced bubble behavior under different ambient pressures. J Appl Phys 120:043302. https://doi.org/ $10.1063 / 1.4959082$

Zhang Z, Wang G, Nie Y, Ji J (2016b) Hydrodynamic cavitation as an efficient method for the formation of sub-100nm O/W emulsions with high stability. Chin J Chem Eng 24:1477-1480. https://doi. org/10.1016/j.cjche.2016.04.011

Zhang Q, Luo J, Zhai Y, Li Y (2018) Improved Instruments and methods for the photographic study of spark-induced cavitation bubbles. Water 10:1683. https://doi.org/10.3390/w10111683

Zhang H, Ren X, Luo C et al (2019) Study on transient characteristics and influencing of temperature on cavitation bubbles in various environments. Optik 187:25-33. https://doi.org/10.1016/j.ijleo. 2019.01.076

Zheng Q, Durben DJ, Wolf GH, Angell CA (1991) Liquids at large negative pressures: water at the homogeneous nucleation limit. Science 254:829-832. https://doi.org/10.1126/science.254.5033.829 
Zupanc M, Kosjek T, Petkovšek M et al (2014) Shear-induced hydrodynamic cavitation as a tool for pharmaceutical micropollutants removal from urban wastewater. Ultrason Sonochem 21:12131221. https://doi.org/10.1016/j.ultsonch.2013.10.025

Zupanc M, Pandur Ž, Stepišnik Perdih T et al (2019) Effects of cavitation on different microorganisms: the current understanding of the mechanisms taking place behind the phenomenon. A review and proposals for further research. Ultrason Sonochem 57:147-165. https://doi.org/10.1016/j.ultsonch.2019.05.009
Zwaan E, Le Gac S, Tsuji K, Ohl C-D (2007) Controlled cavitation in microfluidic systems. Phys Rev Lett 98:254501. https://doi.org/10. 1103/PhysRevLett.98.254501

Publisher's Note Springer Nature remains neutral with regard to jurisdictional claims in published maps and institutional affiliations. 